\title{
EVAluación del fitoplancton en El EMBalse Escaba (TucumÁN - Argentina) PARA CARACTERIZAR LA CALIDAD DE SUS AGUAS
}

\author{
SILVIA N. MARTÍNEZ DE MARCO ${ }^{1,2^{*}}$, BEATRIZ C. TRACANNA ${ }^{2,3}$, SARA C. ISASMENDI², \\ MARIELA DEL C. ALDERETE ${ }^{4}$, MARÍA DE LOS Á. TABOADA ${ }^{1-3}$ y VIRGINIA MIRANDE ${ }^{1,2}$
}

\begin{abstract}
Summary: Assessment of phytoplankton in the Escaba reservoir, Tucumán, Argentina, in order to characterize the water quality. The aim of this study was to analyze space-time variations of phytoplankton in the limnetic zone of the Escaba reservoir and the mouth of its tributary rivers, Chavarría, Las Moras, El Chorro and Singuil, and compare these with physicochemical variables in order to characterize the water quality between 2010 and 2012. One hundred and thirty-five taxa were determined: 77 Bacillariophyceae, 40 Chlorophyta, 12 Cyanophyta, 5 Euglenophyta and 1 Dinophyta. Algal density varied between 84 and 6,924 individuals/ml with Ceratium hirundinella as the dominant species, except for spring 2011. Chlorophyll-a varied seasonally and in space with values between 6 and 2,511 $\mu \mathrm{g} / \mathrm{l}$, with higher numbers for the affluents. Elevated biomass values and the polysaprobic character of the waters defined a deficient or bad ecological status. The specific diversity oscillated between 0 and 2.76. The generalized linear model detected changes in the abundancy of $C$. hirundinella between the two seasons, the sites and months assayed, which were associated with differences in certain physicochemical variables (ammonium, dissolved oxygen (DO) and biological oxygen demand (BOD5)) A negative relationship was only observed between dinoflagellates and ammonium in November 2011. The present study has provided new information regarding the algal biodiversity of the Escaba dam and distribution of $C$. hirundinella in Tucumán province.
\end{abstract}

Key words: Phytoplankton, Ceratium hirundinella, water quality, Escaba reservoir, Tucumán, Argentina.

\begin{abstract}
Resumen: El objetivo de este trabajo es analizar las variaciones espacio-temporales del fitoplancton de la zona limnética del embalse Escaba y la desembocadura de sus tributarios: ríos Chavarría, Las Moras, El Chorro y Singuil, respecto a variables fisicoquímicas para caracterizar la calidad del agua durante 2010-2012. Se determinaron 135 taxones: Bacillariophyceae (77), Chlorophyta (40), Cyanophyta (12), Euglenophyta (5) y Dinophyta (1). La densidad algal varió de 84 a $6924 \mathrm{ind} / \mathrm{ml}$ y Ceratium hirundinella fue dominante excepto en primavera de 2011. La clorofila a tuvo un rango variable a nivel temporal y espacial fluctuando entre 6 y $2511 \mu \mathrm{g} / \mathrm{l}$, encontrándose mayores registros en los afluentes. Los elevados valores de biomasa y la caracterización polisapróbica de sus aguas determinaron un estado ecológico deficiente o malo. La diversidad específica osciló entre 0-2,76. El modelo lineal generalizado detectó cambios en las abundancias de C. hirundinella entre las dos temporadas, sitios y meses acompañados por diferencias en algunas variables fisicoquímicas (amonio, oxígeno disuelto (OD) y demanda biológica de oxígeno (DBO5)) Sólo se observó durante noviembre de 2011 una relación inversa entre la densidad del dinoflagelado con el amonio. Este trabajo aportó al conocimiento de la biodiversidad algal del embalse Escaba y amplió la distribución de $C$. hirundinella en la provincia de Tucumán.
\end{abstract}

Palabras clave: Fitoplancton, Ceratium hirundinella, calidad de las aguas, embalse Escaba, Tucumán, Argentina.

\footnotetext{
${ }^{1}$ Instituto de Ficología (IFico), Fundación Miguel Lillo. Miguel Lillo 251, T4000JFE, San Miguel de Tucumán, Argentina

2 Instituto de Limnología del Noroeste Argentino (ILINOA), Facultad de Ciencias Naturales e IML, Universidad Nacional de Tucumán (UNT)

${ }^{3}$ Unidad Ejecutora Lillo (UEL), CONICET

${ }^{4}$ Cátedra de Ecología General, Facultad de Ciencias Naturales e IML, UNT

* silmdemar@gmail.com
} 


\section{INTRODUCCIÓN}

Los embalses son ecosistemas artificiales creados por el hombre y su construcción se realiza con distintos fines: generación de energía eléctrica, provisión de agua potable, regulación de crecidas, turismo, etc. (Navarro et al., 2010). Sin embargo, es necesario supervisar y evaluar los impactos en la calidad del agua a fin de comprender las interacciones que ocurren entre los usos de la cuenca de drenaje y la preservación o deterioro del cuerpo de agua (Tundisi, 2008).

El manejo de lagos y embalses creció de manera destacada con el uso de asociaciones de especies como indicadoras de niveles tróficos (Huszar et al., 1998). La abundancia de especies fitoplanctónicas y la estructura de esta taxocenosis varían a lo largo de gradientes tróficos y estacionales según numerosos autores (Rott, 1988; del Giorgio et al., 1991; Seip \& Reynolds, 1995; Rojo, 1998). La eutrofización ocasiona un incremento y una alteración en la composición del fitoplancton (Reynolds, 1998; Western, 2001) en la que influyen, entre otros factores, las altas temperaturas, diferencias en la estabilidad térmica y circulación de nutrientes.

En las últimas décadas se ha incrementado significativamente el interés por la ecología de la ficoflora de los embalses debido a que un desarrollo excesivo de algunas especies puede limitar su uso recreativo como así también afectar su calidad estética, entre otros efectos (Rodríguez et al., 2000). La utilización de índices biológicos en los estudios realizados por más de dos décadas en tres ambientes leníticos de la provincia de Tucumán (C. Gelsi, Río Hondo y La Angostura) resultó ser una herramienta útil para la caracterización de la calidad del agua de estos reservorios (Tracanna et al. 2014a). Durante los años 2004 y 2008 en el embalse Escaba, de acuerdo a información periodística de un diario local, se produjeron episodios con una gran mortandad de peces por falta de oxígeno debido a un marcado descenso del nivel de agua, aumento de nutrientes y crecimiento algal. Estos eventos motivaron el inicio de un estudio limnológico a partir de 2010 para determinar los efectos que producían las distintas actividades en el reservorio. Entre los antecedentes se puede mencionar el análisis de las variables físicas y químicas publicado en Tracanna et al. (2014b), donde se concluye que las fluctuaciones hidrométricas anuales coincidieron con la estacionalidad pluvial que determina dos períodos bien definidos de aguas altas (verano-otoño) y aguas bajas (inviernoprimavera). Los máximos valores de transparencia concuerdan con el período de estiaje y el reservorio se caracterizó de acuerdo a Margalef (1983) como monomíctico cálido con estratificación térmica en primavera-verano, con mineralización débil y agua de tipo bicarbonatada-cálcica-sódica. En la zona limnética durante los meses cálidos, la concentración de oxígeno tuvo un gradiente mayor en el epilimnion y déficit en los estratos profundos sin alcanzar condiciones anóxicas, coincidente con la estratificación del embalse mientras que en el período de mezcla el oxígeno se mantuvo constante en la columna de agua. La mayoría de los registros de demanda biológica de oxígeno (DBO5) detectados en las desembocaduras de los ríos y en la zona limnética fueron superiores a $8 \mathrm{mg} \mathrm{O}_{2} / 1$. En general, el fósforo se comportó como limitante y el ecosistema estudiado se determinó como hipertrófico considerando el nitrógeno y fósforo totales y transparencia de acuerdo a la clasificación trófica propuesta en Conzonno (2009).

Elobjetivo de este trabajo es analizar las variaciones espacio-temporales del fitoplancton respecto a variables fisicoquímicas, para caracterizar la calidad del agua del embalse Escaba y desembocadura de sus tributarios durante 2010-2012.

\section{Área de estudio}

El embalse Escaba se encuentra localizado en el departamento Juan Bautista Alberdi, a 110 km al sur de la capital de la provincia. Es el cuarto reservorio de Tucumán en relación a sus dimensiones, su área es de aproximadamente 541 ha, tiene un volumen de $138 \mathrm{hm}^{3}$, una profundidad de $65 \mathrm{~m}$ al momento del llenado y está situado a $650 \mathrm{~m}$ snm (Locascio de Mitrovich et al., 1997; Fernández et al., 2007). El sistema hidrográfico está representado por una de las cuencas más importantes de Tucumán, la del río Marapa, que recibe principalmente el aporte del embalse arriba mencionado y forma parte de la cuenca del río Salí-Dulce. En el embalse Escaba desaguan dos ríos de régimen permanente, Chavarría al norte y Singuil al sur y dos de caudales menores, Las Moras y El Chorro al oeste del espejo de agua (Fernández et al., 2007). Este fue el primer reservorio construido en la provincia en 1940 y se creó para: atenuación de crecidas, riego, producción de energía hidroeléctrica y además para la recreación y promoción turística (Rearte, 1981). 


\section{S. N. Martínez de Marco et al. - Fitoplancton del embalse Escaba}

La litología que se observa en los alrededores se caracteriza por areniscas tobáceas que en algunos sectores pueden presentar clastos de piedra pómez y tufitas finas alternando con bancos de toba (Fernández et al., 2007).

Anualmente el embalse tiene fluctuaciones hidrométricas cíclicas, en el período comprendido entre los meses de mayo-enero, el nivel del agua desciende desde la cota máxima $(631,75 \mathrm{~m})$ a más de $30,00 \mathrm{~m}$ promedio, aumentando a partir de la segunda quincena de enero hasta alcanzar nuevamente el valor máximo. Esta fluctuación se corresponde con la marcada estación seca de la provincia (Pantorrilla \& Núñez Regueiro, 2006).

El clima es templado, con inviernos secos no rigurosos y veranos cálidos, húmedos y lluviosos. Las precipitaciones medias anuales son de 1200 $\mathrm{mm}$, el mes de mayor registro pluvial promedio es febrero $(167 \mathrm{~mm})$, el de menor es agosto $(3 \mathrm{~mm})$ y la temperatura media anual es de $17,6^{\circ} \mathrm{C}$. La posición de los cordones montañosos hace que los vientos del este y sudeste descarguen su humedad del lado tucumano y pasen secos hacia Catamarca (Santillán de Andrés \& Ricci, 1980).

El área de estudio se localiza en el pedemonte de las Sierras Subandinas, zona caracterizada por la Eco-región de las Yungas. En las cercanías, especialmente de los ríos Chavarría y Singuil, la vegetación original ha sido modificada por diversas actividades antrópicas, tales como cultivos de tabaco, citrus y caña de azúcar, obtención de leña y pastoreo de ganado vacuno y caprino.

Actualmente la actividad ganadera es uno de los principales problemas ecológicos, ya que deteriora paulatinamente la vegetación del sotobosque y permite la introducción, en general, de especies chaqueñas (Sirombra, 2003).

\section{Materiales y Métodos}

Se realizaron ocho muestreos estacionales desde agosto de 2010 a mayo de 2012 en la zona más profunda del embalse Escaba (zona limnética) y en la desembocadura de sus cuatro tributarios. En la figura 1 se señala la ubicación de los sitios relevados los cuales fueron georreferenciados mediante GPS (48 Garmin): 1- río Chavarría (2738,803' S; 65²47,229' O), 640 m snm; 2- río Las Moras $\left(27^{\circ} 39,020^{\prime} \mathrm{S} ; 65^{\circ} 47,197^{\prime} \mathrm{O}\right), 617 \mathrm{~m}$ snm; 3- río El Chorro (27³9,984' S; 6546,992'
O), $632 \mathrm{~m} \mathrm{snm}$; 4- río Singuil $\left(27^{\circ} 40,631^{\prime} \mathrm{S}\right.$; 65\%47,102' O), $630 \mathrm{~m}$ snm; 5- zona limnética $\left(27^{\circ} 39,565^{\prime} \mathrm{S} ; 65^{\circ} 45,954^{\prime} \mathrm{O}\right), 642 \mathrm{~m}$ snm. En este último punto se consideraron cuatro niveles: subsuperficial $(20 \mathrm{~cm})$, zona fótica (visibilidad del disco de Secchi), $10 \mathrm{~m}$ y fondo (cuya profundidad varió según la cota). Se debe aclarar que los bajos registros hidrométricos en los meses de noviembre de 2010 y 2011 no permitieron la recolección en la desembocadura del río Las Moras.

La metodología y los resultados de las variables físicas y químicas del agua del embalse se encuentran publicados en Tracanna et al. (2014b).

Las muestras cualitativas del fitoplancton se tomaron con red de $20 \mu \mathrm{m}$ de malla, mediante barridos horizontales subsuperficiales y las cuantitativas se extrajeron directamente de la subsuperficie y estratificadamente en recipientes plásticos de $250 \mathrm{ml}$ y se fijaron in situ. Las colectas fitoplanctónicas correspondientes a las diferentes profundidades de la zona limnética, se hicieron con una trampa Schindler-Patalas. Las determinaciones de la riqueza específica y el recuento de la abundancia algal se efectuaron mediante microscopios ópticos (Leitz con dispositivo de dibujo y Leica DM LS2 y con cámara digital) e invertido (Zeiss IDO2 - 400X), respectivamente. Los análisis cuantitativos se hicieron de acuerdo a la metodología de Utermöhl (1958). Se utilizaron cámaras de sedimentación de $10 \mathrm{ml}$ para la mayor parte de las muestras, sin embargo en algunos ríos se debió emplear de 2 $\mathrm{ml}$ debido a la excesiva cantidad de individuos. Las muestras fueron depositadas en la Colección Ficológica del Herbario Criptogámico de la Fundación Miguel Lillo con la numeración de LIL 22.000 al 22.186.

Para jerarquizar la dominancia de las especies fitoplanctónicas se hizo un diagrama de OlmsteadTukey. Las especies dominantes, constantes, ocasionales y raras se determinaron a partir de la relación entre las densidades de los organismos y sus frecuencias de aparición. Para ello, en la confección del gráfico se tuvieron en cuenta las medias de la frecuencia de aparición relativa y del logaritmo de la densidad absoluta (Sokal \& Rohlf, 1981).

En la determinación de la biomasa (expresada como $\mu \mathrm{g}$ de clorofila $a / 1)$ se siguió la metodología propuesta por Loez (1995) para la extracción de muestras, procesamiento y cálculo. 


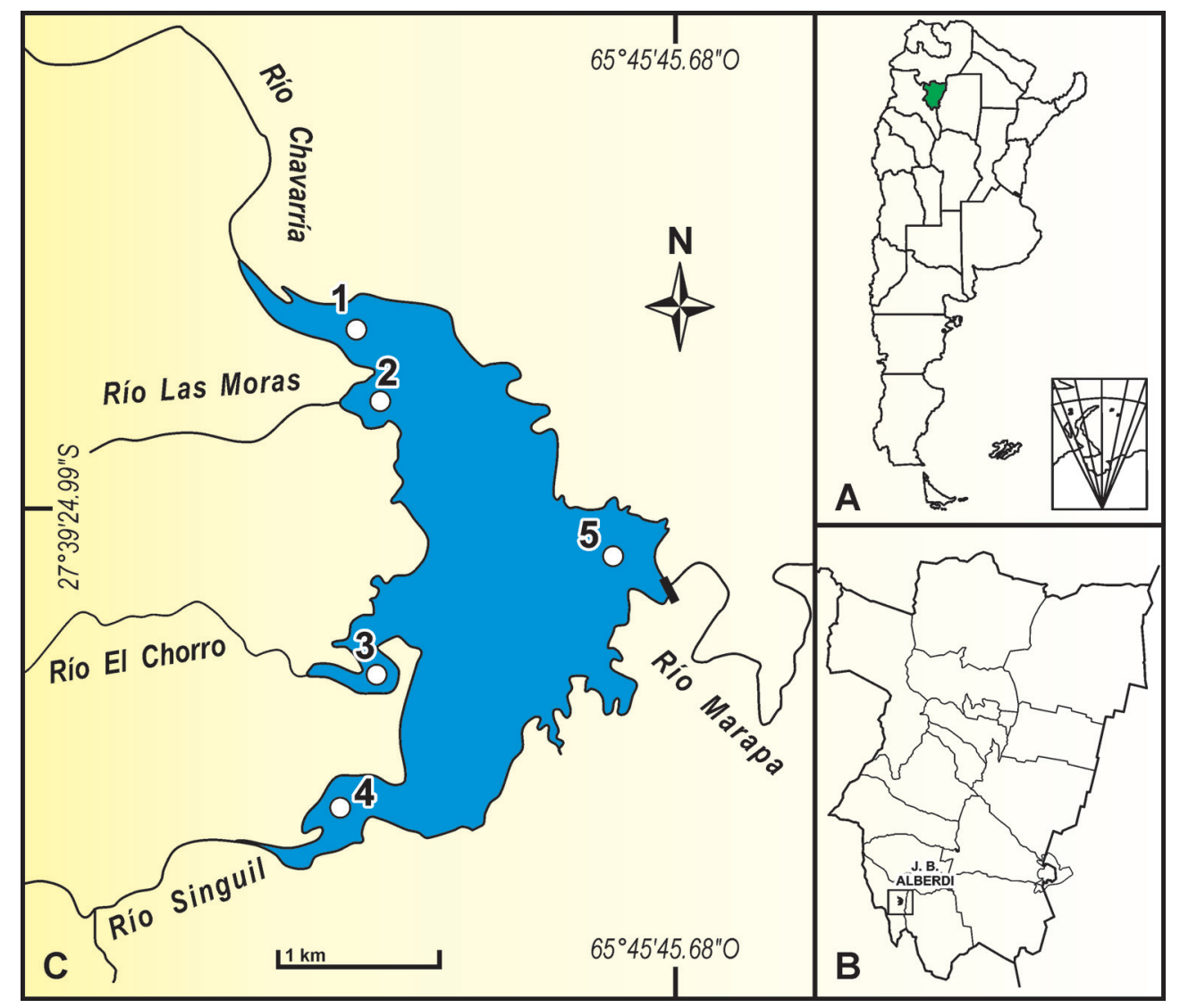

Fig. 1. A: Ubicación de la provincia de Tucumán, Argentina. B: Embalse Escaba en Tucumán. C: Área de estudio y sitios de muestreo: 1- R. Chavarría; 2- R. Las Moras; 3- R. El Chorro; 4- R. Singuil; 5- Zona limnética.

La diversidad fitoplanctónica se estimó aplicando el índice de Shannon-Weaver (Magurran, 2004). El índice y los intervalos de confianza con la técnica bootstrap fueron obtenidos mediante el programa Infostat (Di Rienzo et al., 2013).

En la evaluación de la calidad del agua se empleó el índice sapróbico (IS) de Pantle \& Buck (1955) según el grado sapróbico que considera el comportamiento regional de las especies fitoplanctónicas teniendo en cuenta la $\mathrm{DBO}_{5}$ (Hamm, 1969).

Además, se efectuó un análisis de correlación (coeficiente de Spearman: $\mathrm{p}<0,05 *$ y $\mathrm{p}<0,01 * *$ ) entre las variables físicas, químicas y biológicas, mediante el uso del programa Infostat (Di Rienzo et al., 2013).

Se aplicó el modelo lineal generalizado (MLG) que permitió relacionar una variable respuesta y variables explicativas mediante el uso de otras funciones de errores debido a que la distribución no fue normal (McCullagh \& Nelder, 1983). En este caso, como en los recuentos de $C$. hirundinella se detectó una sobredispersión (alta variabilidad expresada como mayor varianza que la media), los errores aleatorios fueron ajustados a una distribución binomial negativa. Entre varios modelos elaborados donde se relacionó la variable respuesta (abundancia de $C$. hirundinella) y las variables explicativas (temporales (temporada 1: agosto 2010-mayo 2011; temporada 2: agosto 2011-mayo 2012) y meses), sitios de muestreo y fisicoquímicas) se escogió aquel que presentó el menor valor de AIC (criterio de información de análisis) (Akaike, 1973). Los parámetros fisicoquímicos (OD, $\mathrm{DBO}_{5}$, conductividad eléctrica (CE), ortofosfato, $\mathrm{NO}_{3}^{-}, \mathrm{NO}_{2}^{-}, \mathrm{NH}_{4}^{+}$) seleccionados para el modelo tuvieron correlaciones menores al $75 \%$ para evitar multicolinealidad (Coeficiente de Correlación de 


\section{S. N. Martínez de Marco et al. - Fitoplancton del embalse Escaba}

Pearson). Los análisis fueron realizados a través del paquete MASS correspondiente al software R 3.4 (R Development Core Team, 2005). Para interpretar los coeficientes de parámetros estimados en el MLG binomial negativo se utilizaron las relaciones de las variables del modelo matemático examinando el porcentaje de cambio (PC) en las cantidades esperadas, el cual se define como $P C=100 \times[\exp (b)-1]$, donde $b$ es el coeficiente de regresión binomial negativo estimado. El modelo señala el porcentaje de cambio esperado en la variable respuesta por una unidad de cambio en la variable predictora o explicativa manteniendo a todas las otras predictoras constantes (Beaujean \& Morgan, 2016).

\section{Resultados}

Se registraron un total de 127 especies, 7 variedades y 2 formas (Tabla 1) pertenecientes a las Divisiones: Chlorophyta (40), Cyanophyta (12), Euglenophyta (5), Dinophyta (1) y a la Clase Bacillariophyceae (77). Se observaron 54 taxones comunes a todos los sitios, mientras que fueron exclusivos de la zona limnética 21 y de la desembocadura de los tributarios 61. La Tabla 2 muestra los valores mínimos que fluctuaron entre 2 y 12 especies y los máximos que oscilaron entre 21 y 56 taxones.

Las diatomeas, en general, sobresalieron por su riqueza en todas las zonas muestreadas excepto en el río El Chorro (mayo y noviembre de 2011) y en los tres primeros niveles de la zona limnética (noviembre de 2011) donde se destacaron las algas verdes. En la diatomoflora predominaron las pennadas con 28 taxones, especialmente en las desembocaduras, y las céntricas estuvieron representadas por 7 especies. En la primavera de 2011 las clorofitas alcanzaron el mayor número de taxones entre 12 y 20 , mientras que en el resto de los meses su presencia fluctuó entre 1 y 6 .

Los euglenoides fueron registrados en el río Las Moras durante todo el período muestreado y en las desembocaduras de los otros ríos sólo se observaron en agosto de 2010 y/o noviembre de 2011 y en la zona limnética (subsuperficie) en la primavera de 2011 .

Las algas azules estuvieron presentes con bajo número de taxones, hasta 4 en la zona más profunda del embalse. Las especies más frecuentes fueron: Ceratium hirundinella (O. Müller) Dujardin (98\%), Aulacoseira granulata (Ehrenberg) Simonsen (90\%), Ulnaria ulna (Nitzsch) Comperè (56\%), Melosira varians C. Agardh (47\%), Cocconeis placentula Ehrenberg (40\%), A. granulata var. angustissima (O. Müller) Simonsen (37\%), Diatoma vulgaris Bory (37\%) y Botryococcus braunii Kützing (34\%).

En la figura 2 se observa que la riqueza específica mostró una variación temporal similar entre los sitios. En noviembre de 2011 se destaca un notable aumento de taxones en los ríos Singuil, Chavarría y en el fondo de la zona limnética, esta última en general tuvo los máximos valores durante el período estudiado. Los géneros que aportaron el mayor número de especies correspondieron a: Nitzschia y Pinnularia (8), Scenedesmus (7), Gomphonema (5) y Pediastrum (4).

La abundancia fitoplanctónica del embalse Escaba estuvo representada por 78 taxones (Tabla 3). En la figura 3A-E se observa que $C$. hirundinella fue dominante en todo el cuerpo léntico, excepto en la primavera de 2011 cuando el registro de esta Dinophyta fue escaso en la zona limnética con sólo 1-3 ind/ml y nulo en las desembocaduras. La máxima densidad de C. hirundinella se obtuvo en el río Singuil en marzo de 2011 con $6922 \mathrm{ind} / \mathrm{ml}$. En primavera de 2011 las Chlorophyta predominaron sobre todos los grupos algales. En la zona limnética sobresalieron Monoraphidium pusillum (Printz) Komárková-Legnorová (586 ind/ml), Uva sp. (328 ind/ml), Tetradesmus obliquus (Turpin) M. J. Wynne (160 ind $/ \mathrm{ml}$ ), Chlamydomonas globosa J. W. Snow (83 ind $/ \mathrm{ml}$ ) y Desmodesmus spinosus (Chodat) E. H. Hegewald (66 ind/ml). También en las desembocaduras dominaron las especies de algas verdes, en el caso del Chavarría con Uva sp. (361 ind $/ \mathrm{ml})$, Oocystis sp. (25 ind $/ \mathrm{ml})$ y Scenedesmus ecornis (Ehrenberg) Chodat (21 ind/ $\mathrm{ml})$. En el río El Chorro se destacaron Oocystis sp., Chlorococcum sp., Uva sp. y Monoraphidium sp., con valores máximos de 776, 218, 183 y $73 \mathrm{ind} / \mathrm{ml}$, respectivamente y en el Singuil predominaron $U v a$ sp. con $468 \mathrm{ind} / \mathrm{ml}$ seguida por Oocystis sp. con $184 \mathrm{ind} / \mathrm{ml}$ y Monoraphidium sp. con $47 \mathrm{ind} / \mathrm{ml}$. Las diatomeas, en general, estuvieron representadas por un número menor de individuos, alcanzando un máximo de 159 ind/ml en el río Las Moras durante el invierno de 
Bol. Soc. Argent. Bot. 53 (4) 2018

Tabla 1. Fitoplancton del embalse Escaba y de la desembocadura de sus ríos tributarios: Chavarría (RCH), Las Moras (RLM), El Chorro (RECH), Singuil (RS), Zona Limnética (ZL) en el período 2010-2012.

\begin{tabular}{|c|c|c|c|c|c|}
\hline & $\mathrm{RCH}$ & RLM & RECH & RS & ZL \\
\hline \multicolumn{6}{|l|}{ División Cyanophyta } \\
\hline \multicolumn{6}{|l|}{ Clase Cyanophyceae } \\
\hline Leptolyngbya foveolarum (Rabenhorst ex Gomont) Anagnostidis \& Komárek & & & & $\mathrm{x}$ & $\mathrm{X}$ \\
\hline Limnothrix redekei (Goor) Meffert & & & & & $\mathrm{x}$ \\
\hline Lyngbya sp. & $\mathrm{x}$ & & & $x$ & \\
\hline Merismopedia glauca (Ehrenberg) Kützing & & $x$ & & & \\
\hline Merismopedia sp. & & & $\mathrm{x}$ & & \\
\hline Oscillatoria sp. & $x$ & $x$ & & $x$ & \\
\hline Phormidium allorgei (Frémy) Anagnostidis \& Komárek & & $x$ & & & $\mathrm{x}$ \\
\hline P. puteale (Montagne ex Gomont) Anagnostidis \& Komárek & & & & & $\mathrm{x}$ \\
\hline P. subfuscum Kützing ex Gomont & & & & & $\mathrm{x}$ \\
\hline Phormidium sp. & & & & $x$ & \\
\hline Planktolyngbya subtilis (West) Anagnostidis \& Komárek & & & & & $\mathrm{x}$ \\
\hline Pseudanabaena sp. & & & & $x$ & \\
\hline \multicolumn{6}{|l|}{ División Chlorophyta } \\
\hline Ankistrodesmus fusiformis Corda & $\mathrm{x}$ & & $x$ & $x$ & $x$ \\
\hline Botryococcus braunii Kützing & $\mathrm{X}$ & $\mathrm{X}$ & $\mathrm{x}$ & $x$ & $\mathrm{x}$ \\
\hline Chlamydomonas globosa J. W. Snow & $x$ & & & $x$ & $\mathrm{x}$ \\
\hline Chlorococcum sp. & & & $\mathrm{x}$ & & \\
\hline Closterium leibleinii Kützing ex Ralfs & & & & $x$ & $x$ \\
\hline C. moniliferum var. concavum Klebs & & & & $\mathrm{x}$ & $\mathrm{x}$ \\
\hline Closterium sp. & $x$ & & & $x$ & \\
\hline Cosmarium subtumidum Nordstedt & & & & $x$ & \\
\hline Cosmarium sp. & $\mathrm{x}$ & $\mathrm{x}$ & $\mathrm{x}$ & $x$ & \\
\hline Desmodesmus armatus var. Iongispina (Chodat) E. H. Hegewald & $\mathrm{x}$ & & & & \\
\hline D. spinosus (Chodat) E. H. Hegewald & & & & & $\mathrm{x}$ \\
\hline Eudorina elegans Ehrenberg & $\mathrm{x}$ & $\mathrm{x}$ & $x$ & $\mathrm{x}$ & $\mathrm{x}$ \\
\hline Monoraphidium pusillum (Printz) Komárková-Legnorová & $x$ & & $x$ & $x$ & $x$ \\
\hline Monoraphidium sp. & $\mathrm{X}$ & $\mathrm{x}$ & $x$ & $x$ & \\
\hline Oedogonium sp. & $x$ & & $\mathrm{x}$ & & $\mathrm{x}$ \\
\hline Oocystis solitaria Wittrock & & & $x$ & $x$ & \\
\hline Oocystis sp. & $x$ & $x$ & $x$ & $x$ & \\
\hline Pandorina morum (O. Müller) Bory & & & & & $x$ \\
\hline Pediastrum boryanum (Turpin) Meneghini & & & $x$ & $x$ & $x$ \\
\hline P. boryanum var. brevicorne A. Braun & $\mathrm{X}$ & & & $x$ & \\
\hline P. duplex Meyen & & & $x$ & $x$ & $x$ \\
\hline P. duplex var. punctatum (Willi Krieger) Parra & $\mathrm{x}$ & & & & \\
\hline Pediastrum sp. & & & & & $x$ \\
\hline Scenedesmus acutus f. costulatus (Chodat) Uherkovich & $x$ & & & $\mathrm{x}$ & $\mathrm{x}$ \\
\hline S. arcuatus (Lemmermann) Lemmermann & & & $x$ & & \\
\hline
\end{tabular}




\begin{tabular}{|c|c|c|c|c|c|}
\hline & $\mathrm{RCH}$ & RLM & RECH & RS & ZL \\
\hline S. brevispina (G. M. Smith) Chodat & $\mathrm{X}$ & & & & \\
\hline S. ecornis (Ehrenberg) Chodat & $\mathrm{X}$ & & $\mathrm{X}$ & $\mathrm{X}$ & $\mathrm{X}$ \\
\hline S. ecornis var. disciformes (Chodat) Chodat & & & & & $\mathrm{X}$ \\
\hline S. ovalternus var. graevenitzii (C. Bernard) Chodat & & & $\mathrm{X}$ & & \\
\hline S. quadricauda (Turpin) Brébisson & $\mathrm{X}$ & $\mathrm{x}$ & $\mathrm{X}$ & $\mathrm{X}$ & $\mathrm{X}$ \\
\hline Scenedesmus sp. & $\mathrm{X}$ & & $\mathrm{X}$ & $\mathrm{X}$ & \\
\hline Selenastrum sp. & $\mathrm{X}$ & & $\mathrm{X}$ & & $\mathrm{X}$ \\
\hline Sphaerocystis schroeteri Chodat & $\mathrm{X}$ & & & $\mathrm{X}$ & \\
\hline Spirogyra sp. & $x$ & $\mathrm{x}$ & & $\mathrm{X}$ & $\mathrm{X}$ \\
\hline Stauridium tetras (Ehrenberg) E. Hegewald & $\mathrm{X}$ & & $\mathrm{X}$ & $\mathrm{X}$ & $\mathrm{X}$ \\
\hline Stigeoclonium sp. & $\mathrm{X}$ & & & $\mathrm{X}$ & $\mathrm{X}$ \\
\hline Tetradesmus lagerheimii M. J. Wynne \& Guiry & & & $\mathrm{X}$ & & \\
\hline T. obliquus (Turpin) M. J. Wynne & & & $\mathrm{X}$ & $\mathrm{X}$ & $\mathrm{X}$ \\
\hline Tetraedron sp. & & & & & $\mathrm{X}$ \\
\hline Uva sp. & $\mathrm{X}$ & & $\mathrm{X}$ & $\mathrm{X}$ & $\mathrm{X}$ \\
\hline \multicolumn{6}{|l|}{ División Dinophyta } \\
\hline \multicolumn{6}{|l|}{ Clase Dinophyceae } \\
\hline Ceratium hirundinella & $\mathrm{X}$ & $\mathrm{X}$ & $\mathrm{X}$ & $\mathrm{X}$ & $\mathrm{X}$ \\
\hline \multicolumn{6}{|l|}{ División Heterokontophyta } \\
\hline \multicolumn{6}{|l|}{ Clase Bacillariophyceae } \\
\hline Achnanthes coarctata (Brébisson ex W. Smith) Grunow & & & & $\mathrm{X}$ & \\
\hline Amphipleura lindheimeri Grunow & & $\mathrm{X}$ & & $\mathrm{X}$ & $\mathrm{X}$ \\
\hline Amphora ovalis (Kützing) Kützing & & & & $\mathrm{X}$ & $\mathrm{X}$ \\
\hline Amphora sp. & & & & $\mathrm{X}$ & $\mathrm{X}$ \\
\hline Aulacoseira granulata (Ehrenberg) Simonsen & $\mathrm{X}$ & $\mathrm{X}$ & $\mathrm{X}$ & $\mathrm{X}$ & $\mathrm{X}$ \\
\hline A. granulata var. angustissima (O. Müller) Simonsen & $\mathrm{X}$ & $\mathrm{X}$ & $x$ & $\mathrm{X}$ & $\mathrm{X}$ \\
\hline Caloneis westii (W. Smith) Hendey & & & & $X$ & \\
\hline Cocconeis placentula Ehrenberg & $\mathrm{X}$ & $\mathrm{X}$ & $\mathrm{X}$ & $\mathrm{x}$ & $\mathrm{X}$ \\
\hline Craticula ambigua (Ehrenberg) D. G. Mann & $\mathrm{X}$ & & & $\mathrm{X}$ & $\mathrm{X}$ \\
\hline C. cuspidata (Kützing) D. G. Mann & & & & $\mathrm{X}$ & $\mathrm{X}$ \\
\hline Craticula sp. & $\mathrm{X}$ & & & & \\
\hline Cyclotella meneghiniana Kützing & $\mathrm{X}$ & & $\mathrm{x}$ & $\mathrm{X}$ & $\mathrm{X}$ \\
\hline Cymatopleura solea (Brébisson) W. Smith & & & & $\mathrm{X}$ & \\
\hline Cymbella affinis Kützing & $\mathrm{X}$ & $\mathrm{X}$ & $\mathrm{X}$ & $\mathrm{X}$ & $\mathrm{X}$ \\
\hline C. simonsenii Krammer & & & & $\mathrm{X}$ & \\
\hline C. tumida (Brébisson) Van Heurck & $\mathrm{X}$ & & & & $\mathrm{X}$ \\
\hline Cymbopleura amphicephala (Nägeli) Krammer & & & $\mathrm{X}$ & & $\mathrm{X}$ \\
\hline Diatoma vulgaris Bory & $\mathrm{X}$ & $X$ & $X$ & $\mathrm{X}$ & $\mathrm{X}$ \\
\hline Diploneis boldtiana P. Cleve & & & & $\mathrm{X}$ & \\
\hline D. ovalis (Hilse) Cleve & & & & & $x$ \\
\hline Epithemia argus (Ehrenberg) Kützing & & & & $\mathrm{X}$ & \\
\hline Epithemia sp. & & & & $\mathrm{X}$ & \\
\hline
\end{tabular}


Bol. Soc. Argent. Bot. 53 (4) 2018

\begin{tabular}{|c|c|c|c|c|c|}
\hline & $\mathrm{RCH}$ & RLM & RECH & RS & $\overline{\mathrm{ZL}}$ \\
\hline Eunotia praerrupta Ehrenberg & & & & $\mathrm{x}$ & \\
\hline Eunotia sp. & & & & $\mathrm{x}$ & \\
\hline Fragilaria capucina Desmazières & & & $\mathrm{x}$ & & \\
\hline Fragilaria sp. & $\mathrm{x}$ & & $\mathrm{x}$ & $\mathrm{x}$ & \\
\hline Frustulia vulgaris (Thwaites) De Toni & & & & & $\mathrm{x}$ \\
\hline Frustulia sp. & $x$ & & & & $x$ \\
\hline Gomphonema acuminatum Ehrenberg & & & & $\mathrm{x}$ & \\
\hline G. clavatum Ehrenberg & $\mathrm{x}$ & $\mathrm{x}$ & $\mathrm{x}$ & $\mathrm{x}$ & $\mathrm{x}$ \\
\hline G. olivaceum (Hornemann) Brébisson & & & $\mathrm{x}$ & $\mathrm{x}$ & \\
\hline G. parvulum (Kützing) Kützing & & & & & $\mathrm{x}$ \\
\hline G. truncatum Ehrenberg & $\mathrm{x}$ & & & $\mathrm{x}$ & $\mathrm{x}$ \\
\hline Gomphonema sp. & & $x$ & $x$ & $x$ & $x$ \\
\hline Gyrosigma sp. & & & & $\mathrm{x}$ & $\mathrm{X}$ \\
\hline Hantzschia amphioxys (Ehrenberg) Grunow & & & $\mathrm{x}$ & $\mathrm{x}$ & $\mathrm{x}$ \\
\hline Hyalodiscus sp. & & & & $\mathrm{x}$ & \\
\hline Iconella linearis (W. Smith) Ruck \& Nakov & & & & & $\mathrm{x}$ \\
\hline I. tenera (W. Gregory) Ruck \& Nakov & $\mathrm{x}$ & & & & \\
\hline Lemnicola hungarica (Grunow) Round \& Basson & & $\mathrm{x}$ & & & \\
\hline Luticola dapalis (Frenguelli) D. G. Mann & $\mathrm{x}$ & & & & \\
\hline L. goeppertiana (Bleisch) D. G. Mann & & & & $\mathrm{x}$ & $\mathrm{x}$ \\
\hline L. mutica (Kützing) D. G. Mann & $\mathrm{x}$ & & & & \\
\hline Melosira varians Agardh & $\mathrm{x}$ & $\mathrm{x}$ & $\mathrm{x}$ & $\mathrm{x}$ & $\mathrm{x}$ \\
\hline Navicula amphiceropsis Lange-Bertalot \& U. Rumrich & $\mathrm{x}$ & & $\mathrm{x}$ & $\mathrm{x}$ & \\
\hline N. menisculus Schumann & & & & $\mathrm{x}$ & \\
\hline Navicula sp. & $\mathrm{x}$ & $\mathrm{x}$ & $\mathrm{x}$ & $\mathrm{x}$ & \\
\hline Nitzschia acicularis (Kützing) W. Smith & & & & $x$ & \\
\hline N. gracilis Hantzsch & & & & & $\mathrm{x}$ \\
\hline N. linearis (Agardh) W. Smith & & $\mathrm{x}$ & & & $x$ \\
\hline N. obtusa W. Smith & & & $\mathrm{x}$ & $\mathrm{x}$ & \\
\hline N. palea (Kützing) W. Smith & & $\mathrm{x}$ & & & $\mathrm{X}$ \\
\hline N. sigma (Kützing) W. Smith & $x$ & & & $x$ & \\
\hline N. tryblionella Hantzsch & $x$ & & & & \\
\hline N. vermicularis(Kützing) Hantzsch & & & & & $\mathrm{x}$ \\
\hline Nitzschia spp. & $\mathrm{x}$ & $\mathrm{x}$ & $\mathrm{x}$ & $\mathrm{x}$ & \\
\hline Orthoseira sp. & $\mathrm{x}$ & & & $\mathrm{x}$ & $x$ \\
\hline Pinnularia acrosphaeria W. Smith & $\mathrm{x}$ & & $\mathrm{x}$ & $\mathrm{x}$ & $\mathrm{x}$ \\
\hline P. borealis Ehrenberg & & & $x$ & & \\
\hline P. gibba Ehrenberg & & & & $x$ & $x$ \\
\hline P. gibba var. linearis Hustedt & & & & & $\mathrm{X}$ \\
\hline P. interrupta W. Smith & & & & & $x$ \\
\hline P. maior (Kützing) Rabenhorst & $x$ & & & & \\
\hline P. subcapitata Gregory & & & & & $\mathrm{x}$ \\
\hline
\end{tabular}




\begin{tabular}{|c|c|c|c|c|c|}
\hline & $\mathrm{RCH}$ & RLM & $\mathrm{RECH}$ & RS & ZL \\
\hline P. viridis (Nitzsch) Ehrenberg & $x$ & $x$ & & & $\mathrm{x}$ \\
\hline Pinnularia sp. & $\mathrm{x}$ & & & $\mathrm{x}$ & \\
\hline Placoneis clementis (Grunow) E. J. Cox & & & $\mathrm{x}$ & & \\
\hline Pleurosira laevis (Ehrenberg) Compère & $\mathrm{x}$ & & & & \\
\hline Reimeria uniseriata S. E. Sala, J. M. Guerrero \& M. E. Ferrario & & & & $\mathrm{x}$ & $\mathrm{x}$ \\
\hline Rhoicosphenia abbreviata (Agardh) Lange-Bertalot & $\mathrm{x}$ & & & & $\mathrm{x}$ \\
\hline Rhopalodia gibba (Ehrenberg) O. Müller & & & & $\mathrm{x}$ & $\mathrm{x}$ \\
\hline R. gibberula (Ehrenberg) O. Müller & & & & $\mathrm{x}$ & \\
\hline Sellaphora pupula (Kützing) Mereschkovsky & $\mathrm{x}$ & & $\mathrm{x}$ & & $\mathrm{x}$ \\
\hline Staurosira construens Ehrenberg & & & & & $\mathrm{x}$ \\
\hline Surirella brebissonii Krammer \& Lange-Bertalot & & $\mathrm{x}$ & & & \\
\hline S. ovalis Brébisson & & & & $\mathrm{x}$ & $\mathrm{x}$ \\
\hline Ulnaria ulna (Nitzsch) Compère & $\mathrm{x}$ & $\mathrm{x}$ & $\mathrm{x}$ & $\mathrm{x}$ & $\mathrm{x}$ \\
\hline \multicolumn{6}{|l|}{ Div. Euglenophyta } \\
\hline \multicolumn{6}{|l|}{ Clase Euglenophyceae } \\
\hline Euglenaformis proxima (Dangeard) M. S. Bennett \& Triemer & $\mathrm{x}$ & & & & \\
\hline Euglena sp. & $\mathrm{x}$ & $\mathrm{x}$ & $\mathrm{x}$ & $\mathrm{x}$ & \\
\hline Phacus sp. 1 & $x$ & $x$ & & $x$ & \\
\hline Phacus sp. 2 & & & & & $x$ \\
\hline Trachelomonas sp. & $\mathrm{x}$ & $\mathrm{x}$ & & & \\
\hline
\end{tabular}

Tabla 2. Riqueza específica, densidad y clorofila a en el embalse Escaba (periodo 2010-2012). Abreviaturas: Mín: mínimo; Máx: máximo; Prom: promedio; DE: desviación estándar.

\begin{tabular}{|c|c|c|c|c|c|c|c|c|c|c|c|c|}
\hline \multirow[b]{2}{*}{ Sitios de muestreo } & \multicolumn{4}{|c|}{ Riqueza específica } & \multicolumn{4}{|c|}{ Densidad (ind/ml) } & \multicolumn{4}{|c|}{ Clorofila a $(\mu \mathrm{g} / \mathrm{l})$} \\
\hline & Mín & Máx & Prom & $\mathrm{DE}$ & Mín & Máx & Prom & DE & Mín & Máx & Prom & DE \\
\hline Zona Limnética (Subsuperficial) & 2 & 30 & 12 & 9 & 278 & 1823 & 1010 & 589 & 19 & 714 & 291 & 245 \\
\hline Zona Limnética (Secchi) & 3 & 21 & 8 & 6 & 221 & 4395 & 1541 & 1471 & $s / d$ & $s / d$ & $s / d$ & $s / d$ \\
\hline Zona Limnética (10 m) & 3 & 23 & 9 & 6 & 355 & 2510 & 1118 & 790 & $s / d$ & $s / d$ & $\mathrm{~s} / \mathrm{d}$ & $s / d$ \\
\hline Zona Limnética (Fondo) & 5 & 45 & 24 & 11 & 84 & 1996 & 656 & 729 & 6 & 706 & 182 & 245 \\
\hline Río Chavarría & 2 & 47 & 13 & 16 & 223 & 5760 & 1552 & 1780 & 7 & 304 & 110 & 110 \\
\hline Río Las Moras & 12 & 25 & 17 & 5 & 580 & 1462 & 1018 & 331 & 15 & 1379 & 506 & 653 \\
\hline Río El Chorro & 2 & 23 & 10 & 8 & 724 & 6001 & 2497 & 1946 & 24 & 2511 & 657 & 896 \\
\hline Río Singuil & 3 & 56 & 17 & 19 & 174 & 6924 & 3514 & 2703 & 11 & 1800 & 719 & 657 \\
\hline
\end{tabular}




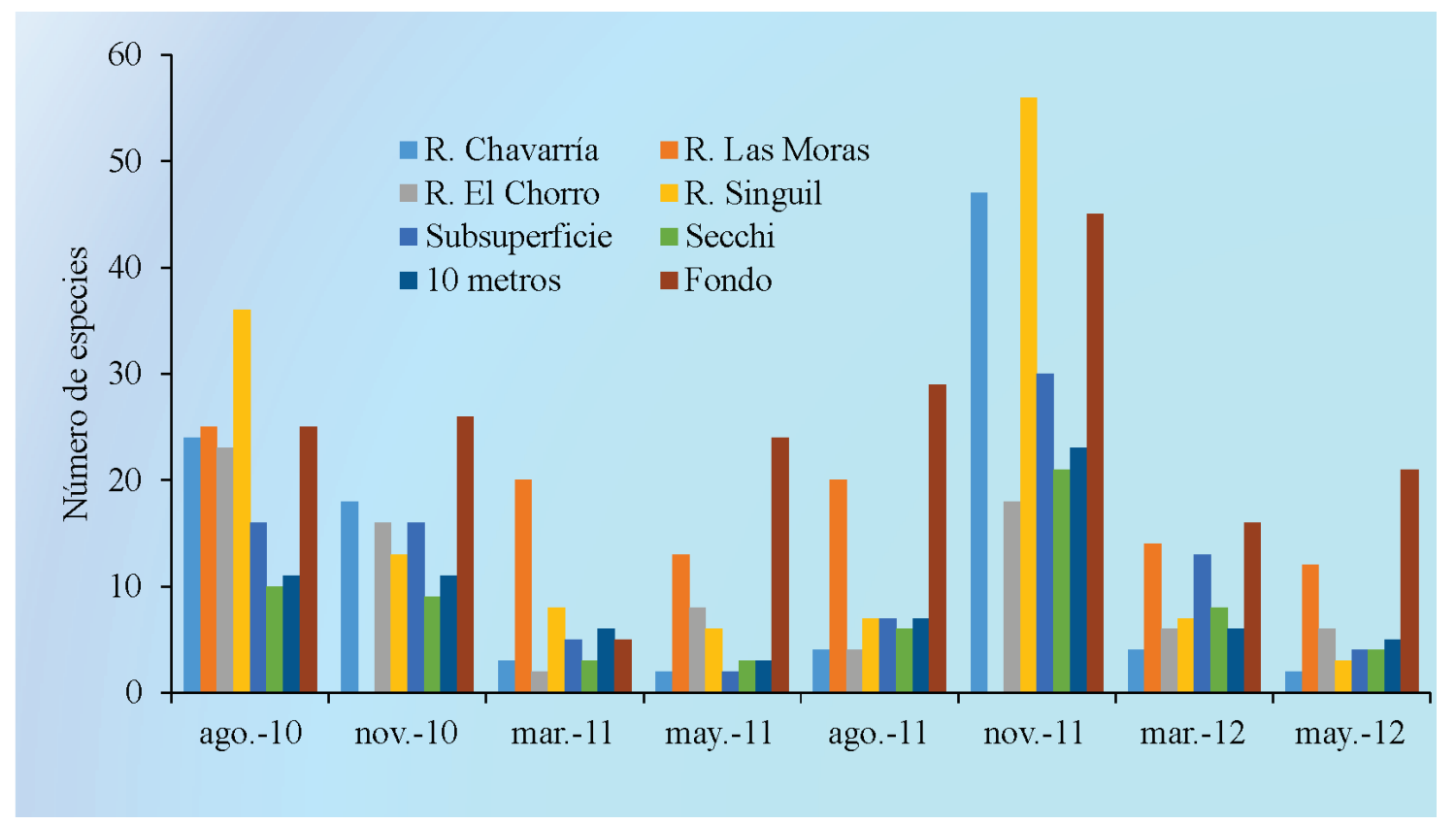

Fig. 2. Fluctuaciones de la riqueza específica fitoplanctónica en los sitios de muestreo del embalse Escaba. ago: agosto, nov: noviembre, mar: marzo, may: mayo. Los niveles que corresponden a la Zona limnética son: Subsuperficial, Secchi, $10 \mathrm{~m}$ y Fondo.

2010 con Pinnularia viridis (Nitzsch) Ehrenberg (30 ind $/ \mathrm{ml})$, U. ulna (19 ind $/ \mathrm{ml})$, D. vulgaris y Nitzschia linearis (Agardh) W. Smith ambas con16 ind $/ \mathrm{ml}$. Las cianobacterias se destacaron especialmente en el fondo de la zona limnética y en el río Las Moras y los euglenoides se cuantificaron solamente en las desembocaduras.

En la Tabla 2 se muestran los valores descriptivos de las variables densidad y clorofila $a$ para el embalse Escaba durante todo el período de estudio. En la zona limnética el máximo de densidad algal obtenido de la suma de las abundancias en toda la columna de agua (10217 ind/ml) ocurrió durante la primavera de 2010, período de aguas bajas y escasa transparencia y fue 2,4 veces mayor que el promedio de los años estudiados (4325 ind/ $\mathrm{ml}$ ). En los muestreos de invierno (aguas bajas) la abundancia fitoplanctónica fue similar en todo el perfil con un total de 986 (agosto de 2010) y $1298 \mathrm{ind} / \mathrm{ml}$ (agosto de 2011). Los mayores registros de densidad algal ocurrieron en general a la profundidad de la lectura del disco de Secchi o a los 10 metros, con una disminución hacia el fondo, a excepción de marzo de 2011 que fue a la inversa (Fig. 4A).
En la figura 4B se aprecia que las fluctuaciones de las densidades del fitoplancton en la desembocadura de los ríos, en general, se comportaron de manera similar disminuyendo en aguas bajas. En el Singuil se registraron los valores mínimo (174 ind/ml, invierno de 2010) y máximos (6922 y $6924 \mathrm{ind} / \mathrm{ml}$ en noviembre de 2010 y marzo de 2011, respectivamente). Las Moras tuvo, generalmente, menores abundancias respecto a los otros tributarios.

En la zona limnética, en el período de mezcla, se registró el mínimo valor de clorofila $a$ en la parte más profunda del embalse con $6 \mu \mathrm{g} / \mathrm{l}$ (agosto de 2010) y el máximo se midió en la subsuperficie con $378 \mu \mathrm{g} / 1$ (mayo de 2012). En períodos estratificados, los datos de biomasa, tanto el menor como el mayor, se obtuvieron en subsuperficie con 19 y $714 \mu \mathrm{g} / 1$ en noviembre y marzo de 2011, respectivamente. En la desembocadura de los cuatro tributarios para agosto de 2010 y noviembre de 2011 se observaron los valores más bajos de clorofila $a$ que fluctuaron entre 7 y $69 \mu \mathrm{g} / \mathrm{l}$. En los ríos Las Moras, El Chorro y Singuil para marzo y mayo de 2011 se dieron los mayores registros de biomasa que variaron 
Tabla 3. Valores máximos de abundancia del fitoplancton del embalse Escaba (ind/ml). Ríos: Chavarría (RCH), Las Moras (RLM), El Chorro (RECH), Singuil (RS), Zona Limnética (subsuperficial (LSU), Secchi (LSE), 10 metros (L10), fondo (LF)). Abr.: Abreviaturas utilizadas en el diagrama de Olmstead-Tukey.

\begin{tabular}{|c|c|c|c|c|c|c|c|c|c|}
\hline \multicolumn{2}{|l|}{ Abr. } & \multirow[t]{2}{*}{$\mathrm{RCH}$} & \multirow[t]{2}{*}{ RLM } & \multirow[t]{2}{*}{ RECH } & \multirow[t]{2}{*}{ RS } & \multirow[t]{2}{*}{ LSU } & \multirow[t]{2}{*}{ LSE } & \multirow[t]{2}{*}{ L10 } & \multirow[t]{2}{*}{ LF } \\
\hline & División Cyanophyta & & & & & & & & \\
\hline & Leptolyngbya foveolarum & & & & & & & & 27 \\
\hline & Lyngbya sp. & 1 & & & & & & & \\
\hline & Merismopedia sp. & & & 1 & & & & & \\
\hline Os & Oscillatoria sp. & & 14 & & & & & & \\
\hline \multirow[t]{2}{*}{$\mathrm{Pa}$} & Phormidium allorgei & & 3 & & & & & & 7 \\
\hline & Phormidium sp. & & & & 2 & & & & \\
\hline \multirow[t]{2}{*}{ Ps } & Planktolyngbya subtilis & & & & & & & & 8 \\
\hline & División Chlorophyta & & & & & & & & \\
\hline Af & Ankistrodesmus fusiformis & & & 10 & 4 & 2 & & 2 & \\
\hline $\mathrm{Bb}$ & Botryococcus braunii & & 3 & & & 2 & & & \\
\hline $\mathrm{Cg}$ & Chlamydomonas globosa & & & & & 83 & 19 & & \\
\hline $\mathrm{Ch}$ & Chlorococcum sp. & & & 218 & & & & & \\
\hline Co & Cosmarium sp. & & 7 & 1 & & & & & \\
\hline Ds & Desmodesmus spinosus & & & & & 1 & 7 & 66 & 7 \\
\hline $\mathrm{Ee}$ & Eudorina elegans & 2 & 11 & 4 & & & & & \\
\hline $\mathrm{Mp}$ & Monoraphidium pusillum & & & & & 18 & 586 & 545 & \\
\hline Mo & Monoraphidium sp. & 4 & & 73 & 47 & & & & \\
\hline Oo & Oocystis solitaria & & & 4 & & & & & \\
\hline \multirow[t]{2}{*}{ Od } & Oocystis sp. & 25 & 13 & 776 & 184 & & & & \\
\hline & Pandorina morum & & & & & 1 & & & \\
\hline \multirow[t]{3}{*}{$\mathrm{Pb}$} & Pediastrum boryanum & & & & & & & & 1 \\
\hline & P. boryanum var. brevicorne & & & & 5 & & & & \\
\hline & Pediastrum sp. & & & & & & 2 & & \\
\hline \multirow[t]{2}{*}{ Sc } & Scenedesmus acutus f. costulatus & 1 & & & 2 & 4 & & & 6 \\
\hline & S. arcuatus & & & 5 & & & & & \\
\hline $\mathrm{Se}$ & S. ecornis & 21 & & 4 & 1 & 9 & & & 3 \\
\hline Sd & S. ecornis var. disciformes & & & & & 17 & 10 & 21 & \\
\hline $\mathrm{Sq}$ & S. quadricauda & 3 & 7 & 22 & 4 & 9 & 14 & & 7 \\
\hline $\mathrm{Sp}$ & Scenedesmus sp. & 2 & & 1 & 3 & & & & \\
\hline SI & Selenastrum sp. & 3 & & 1 & & & & 7 & \\
\hline $\mathrm{Sp}$ & Sphaerocystis schroeteri & 1 & & & 3 & & & & \\
\hline $\mathrm{Sg}$ & Spirogyra sp. & & 7 & & 2 & & & & \\
\hline \multirow[t]{2}{*}{ St } & Stauridium tetras & 1 & & 2 & 1 & & 2 & & \\
\hline & Tetradesmus lagerheimii & & & 3 & & & & & \\
\hline \multirow[t]{2}{*}{ To } & T. obliquus & & & 6 & 9 & 18 & 160 & 50 & 29 \\
\hline & Tetraedron sp. & & & & & & 3 & 3 & \\
\hline \multirow[t]{2}{*}{ Us } & Uva sp. & 361 & & 183 & 468 & 328 & 221 & 41 & 14 \\
\hline & División Dinophyta & & & & & & & & \\
\hline $\mathrm{Ch}$ & Ceratium hirundinella & 5756 & 1441 & 5985 & 6922 & 1804 & 4391 & 2503 & 1908 \\
\hline
\end{tabular}


Bol. Soc. Argent. Bot. 53 (4) 2018

\begin{tabular}{|c|c|c|c|c|c|c|c|c|c|}
\hline \multicolumn{2}{|l|}{ Abr. } & $\mathrm{RCH}$ & RLM & RECH & RS & LSU & LSE & L10 & LF \\
\hline & \multicolumn{9}{|l|}{ División Heterokontophyta } \\
\hline & \multicolumn{9}{|l|}{ Clase Bacillariophyceae } \\
\hline $\mathrm{Al}$ & Amphipleura lindheimeri & & 6 & & & & & & 2 \\
\hline Ao & Amphora ovalis & & & & & & & & 2 \\
\hline $\mathrm{Ag}$ & Aulacoseira granulata & 9 & 11 & 9 & 3 & 9 & 4 & 3 & 17 \\
\hline $\mathrm{Aa}$ & A. granulata var. angustissima & & & 4 & 3 & 4 & & 1 & 19 \\
\hline $\mathrm{Cp}$ & Cocconeis placentula & 1 & 13 & 2 & 3 & 3 & & & 5 \\
\hline $\mathrm{Ca}$ & Craticula ambigua & & & & & 1 & & & \\
\hline \multirow[t]{2}{*}{ Cc } & C. cuspidata & & & & & 1 & & & 2 \\
\hline & Craticula sp. & 3 & & & & & & & \\
\hline $\mathrm{Cm}$ & Cyclotella meneghiniana & 5 & & 2 & 11 & 1 & & & \\
\hline $\mathrm{Cf}$ & Cymbella affinis & & 13 & 3 & & & & & 2 \\
\hline $\mathrm{Ct}$ & C. tumida & & & & & & & & 15 \\
\hline Cy & Cymbopleura amphicephala & & & & & 4 & & & 5 \\
\hline Dv & Diatoma vulgaris & & 16 & & & & & & 3 \\
\hline $\mathrm{Fd}$ & Fragilaria sp. & 2 & & 4 & & & & & \\
\hline \multirow[t]{2}{*}{ Gc } & Gomphonema clavatum & & & & 7 & & & & 2 \\
\hline & G. olivaceum & & & 1 & 11 & & & & \\
\hline Gp & G. parvulum & & & & & 1 & & & 4 \\
\hline Gt & G. truncatum & 2 & & & & & & & 5 \\
\hline Gd & Gomphonema sp. & & 10 & 2 & 1 & 1 & & & 6 \\
\hline $\mathrm{Ha}$ & Hantzschia amphioxys & & & & & & & & 1 \\
\hline Mv & Melosira varians & 4 & 5 & 4 & & & & 2 & 1 \\
\hline $\mathrm{Na}$ & Navicula amphiceropsis & & & 3 & 3 & & & & \\
\hline $\mathrm{Nd}$ & Navicula sp. & & 10 & 4 & 4 & & & & \\
\hline $\mathrm{Ng}$ & Nitzschia gracilis & & & & & 2 & & & 4 \\
\hline $\mathrm{NI}$ & N. linearis & & 16 & & & 1 & & & 1 \\
\hline No & N. obtusa & & & 2 & & & & & \\
\hline \multirow[t]{2}{*}{$\mathrm{Np}$} & N. palea & & 5 & & & 13 & & & 12 \\
\hline & Nitzschia spp. & 4 & 6 & 4 & 4 & & & & \\
\hline Pia & Pinnularia acrosphaeria & & & & & & & & 1 \\
\hline $\mathrm{Pg}$ & P. gibba & & & & & & & & 1 \\
\hline Ps & P. subcapitata & & & & & & & & 1 \\
\hline \multirow[t]{2}{*}{$\mathrm{Pv}$} & $P$. viridis & & 30 & & & & & & \\
\hline & Pinnularia sp. & & & & 1 & & & & \\
\hline $\mathrm{Ra}$ & Rhoicosphenia abbreviata & & & & & & & & 2 \\
\hline Sp & Sellaphora pupula & 3 & & 1 & & & & & 4 \\
\hline Sc & Staurosira construens & & & & & 5 & & & 12 \\
\hline \multirow[t]{2}{*}{ Uu } & Ulnaria ulna & 1 & 19 & & 1 & 3 & 1 & 2 & 7 \\
\hline & Div. Euglenophyta & & & & & & & & \\
\hline $\mathrm{Ed}$ & Euglena sp. & 2 & 9 & 14 & 3 & & & & \\
\hline $\mathrm{Ph}$ & Phacus sp. & & & & 2 & & & & \\
\hline $\operatorname{Tr}$ & Trachelomonas sp. & & 9 & & & & & & \\
\hline
\end{tabular}


S. N. Martínez de Marco et al. - Fitoplancton del embalse Escaba
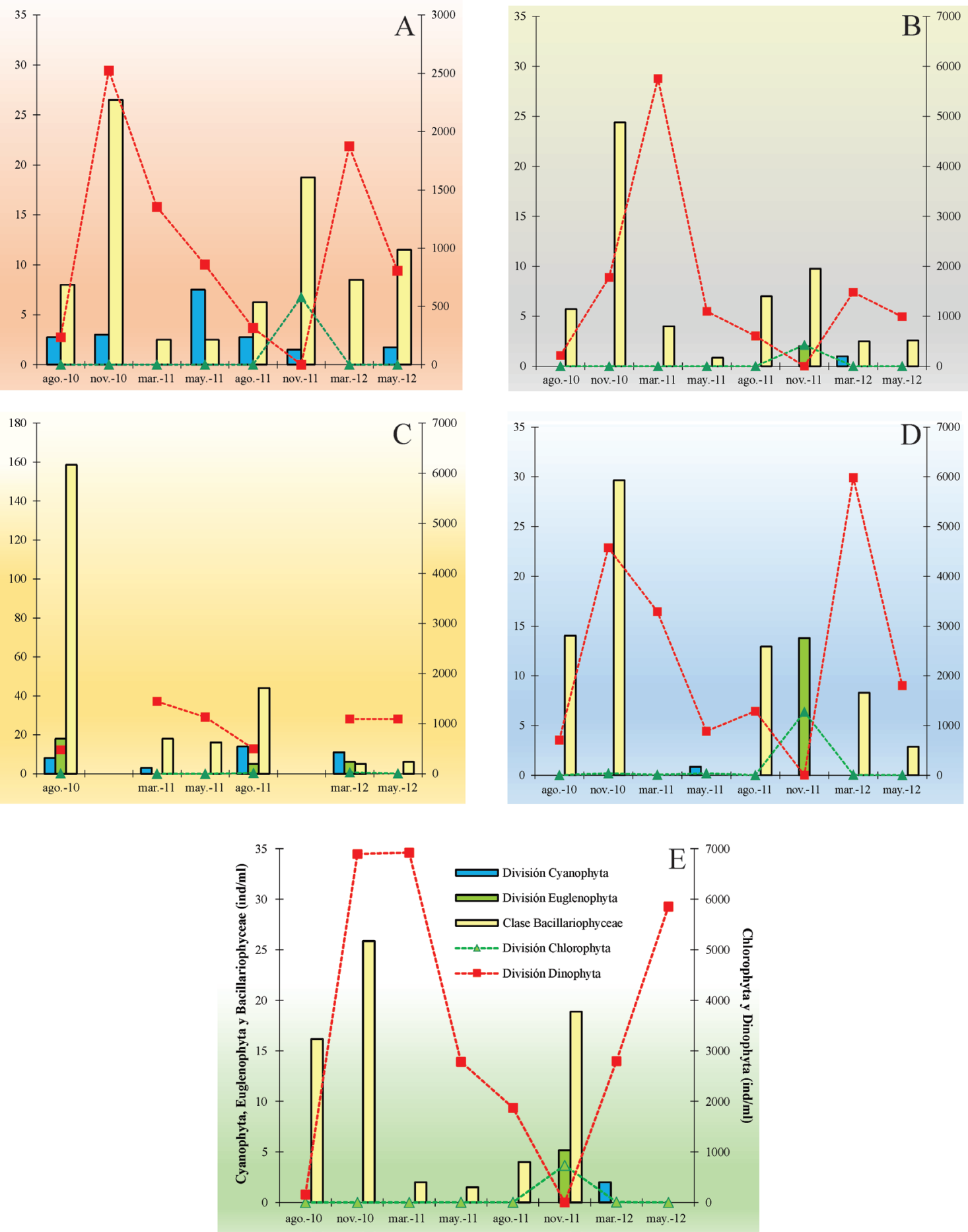

Fig. 3. Variaciones estacionales de las densidades de los grupos fitoplanctónicos encontrados en el embalse Escaba. A: Zona Limnética, B: R. Chavarría, C: R. Las Moras, D: R. El Chorro, E: R. Singuil. ago: agosto, nov: noviembre, mar: marzo, may: mayo. 
Bol. Soc. Argent. Bot. 53 (4) 2018
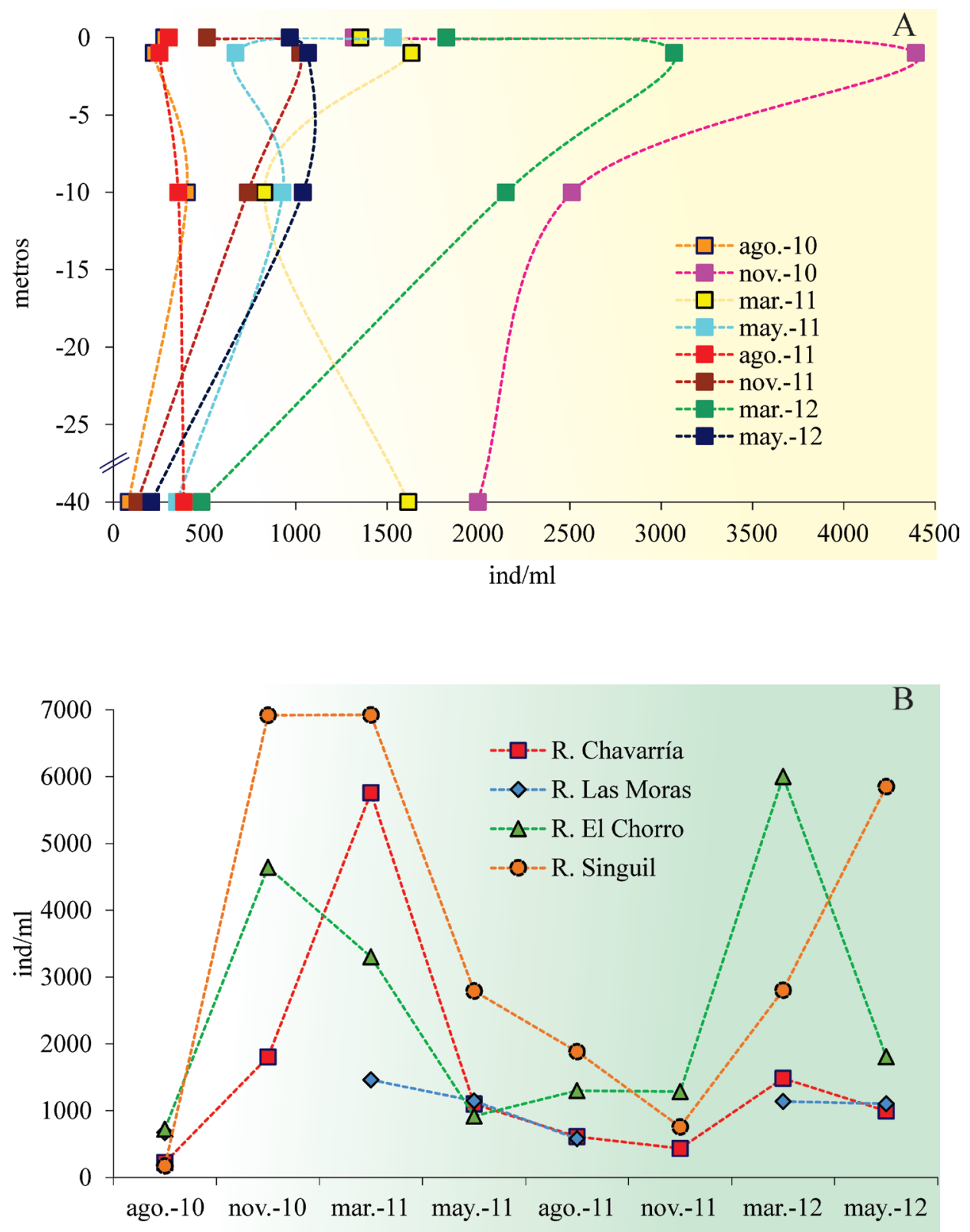

Fig. 4. A: Perfil de las abundancias totales del fitoplancton en los cuatro niveles de la zona limnética del embalse: Subsuperficial, Secchi (0,51-2,40 m), $10 \mathrm{~m}$ y fondo. B: Variaciones de las densidades totales en la desembocadura de los tributarios. ago: agosto, nov: noviembre, mar: marzo, may: mayo.

dentro de un rango de 1279 a $2511 \mu \mathrm{g} / \mathrm{l}$. Las correlaciones significativas de esta variable con las fisicoquímicas y otras biológicas se indican en la Tabla 4.

En el diagrama de Olmstead-Tukey (Fig. 5) se observó que nueve especies fueron dominantes. En esta asociación se destacó $C$. hirundinella, que fue la más abundante y frecuente, acompañada por A. granulata, U. ulna, C. placentula, A. granulata var. angustissima, M. varians, Nitzschia palea (Kützing) W. Smith, D. vulgaris y Scenedesmus quadricauda (Turpin) Brébisson. Fueron constantes tres diatomeas (Cymbopleura amphicephala (Nägeli) Krammer, Gomphonema 
S. N. Martínez de Marco et al. - Fitoplancton del embalse Escaba

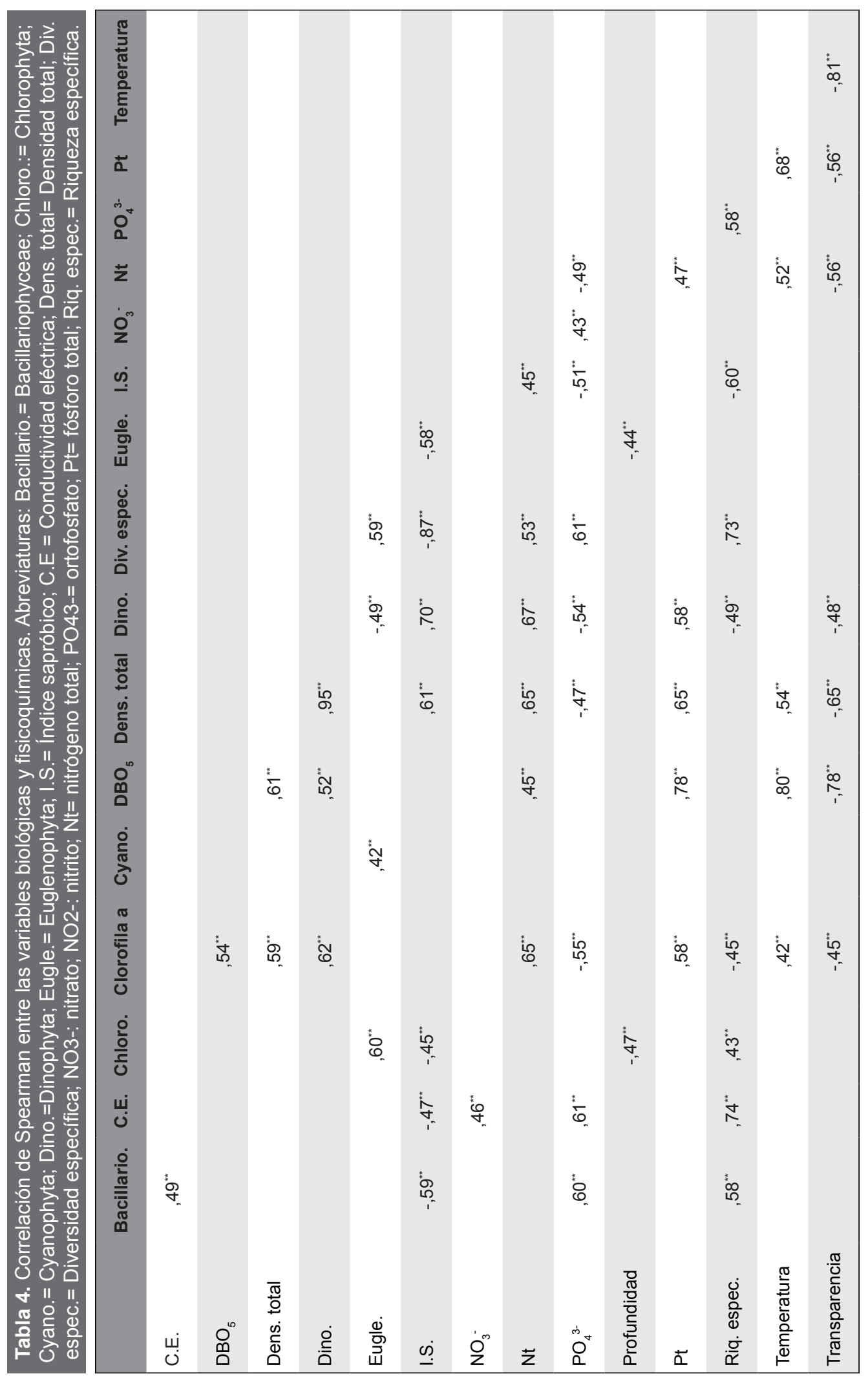




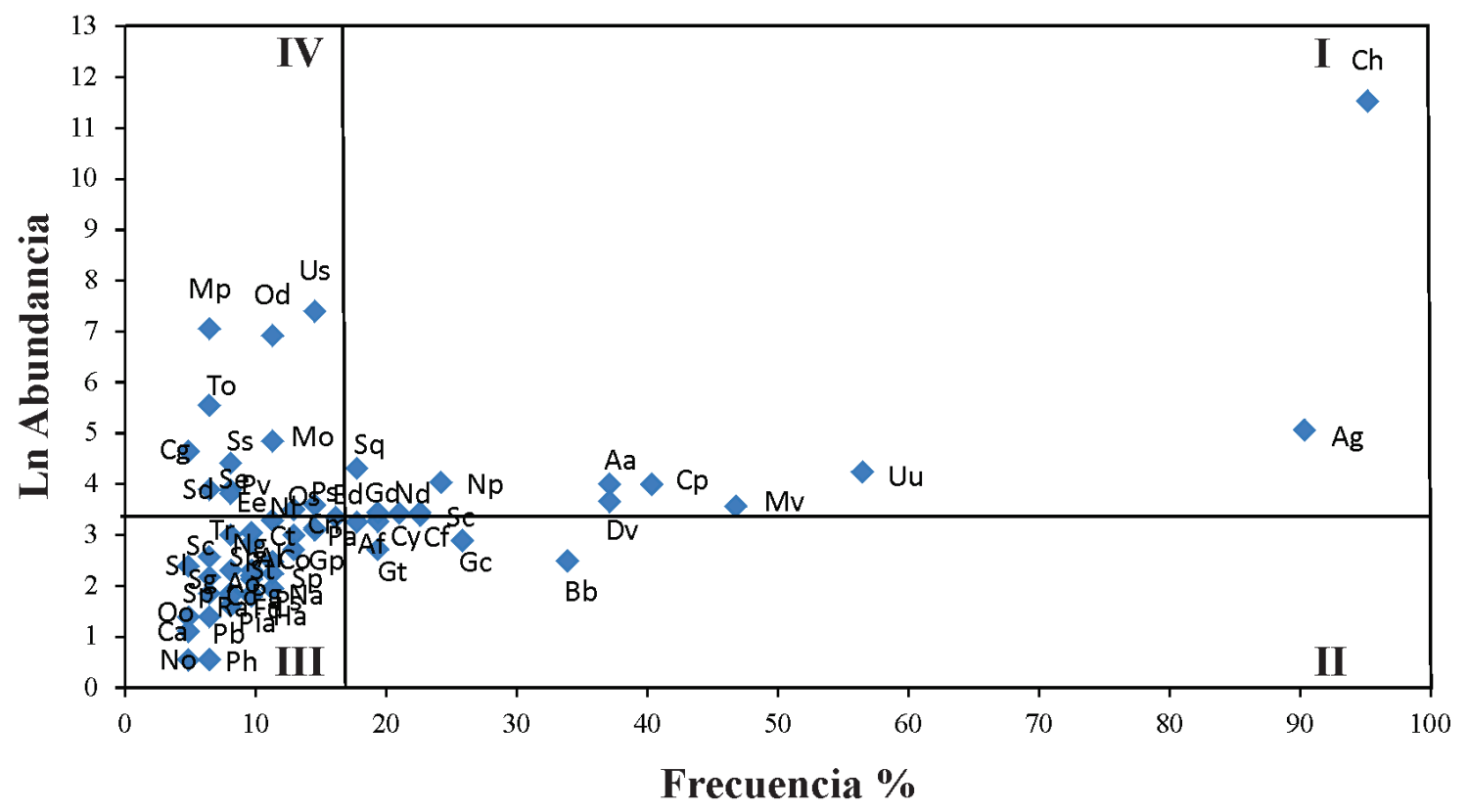

Fig. 5. Diagrama de Olmstead-Tukey del fitoplancton del embalse Escaba. Especies: I dominantes, II constantes, III raras, IV ocasionales.

clavatum Ehrenberg y G. truncatum Ehrenberg) $\mathrm{y}$ dos algas verdes (B. braunii Kützing y Ankistrodesmus fusiformis Corda). Las especies ocasionales estuvieron representadas por: Uva sp., Oocystis sp., M. pusillum, Monoraphidium sp., C. globosa, tres especies de Scenedesmus, D. spinosus, P. viridis, P. subcapitata Gregory y Oscillatoria sp. Las especies raras fueron 37, la mayoría diatomeas y algas verdes.

Los valores del índice de diversidad de ShannonWeaver con sus intervalos de confianza para cada sitio durante todos los muestreos se muestran en la Tabla 5. A pesar de que los resultados de diversidad fueron bajos se observaron diferencias espaciales y temporales. En todos los niveles de la zona limnética se registraron los máximos valores de diversidad durante la primavera de 2011, destacándose el mayor de 2,76 en profundidad. Con respecto a los tributarios, el máximo de 1,38 correspondió a Las Moras en invierno de 2010, mientras que en las otras desembocaduras se registraron en primavera de 2011.

De acuerdo al índice sapróbico las aguas de la zona limnética y desembocadura de los tributarios fueron caracterizadas como polisapróbicas $(3,5-4,0)$ salvo en noviembre de 2011 que fueron $\alpha$-mesosapróbicas $(2,5-3,0)$. Por consiguiente, la calidad del agua pudo definirse como de "contaminación muy fuerte" excepto en primavera de 2011 en que se determinó como de "contaminación fuerte".

El modelo lineal generalizado seleccionado con el menor valor de AIC detectó cambios en las abundancias de C. hirundinella entre las dos temporadas analizadas, sitios y meses acompañados por diferencias en algunas variables fisicoquímicas (Tabla 6). Así, las abundancias se relacionaron negativamente con el río Chavarría (48\% de decrecimiento) pero positivamente con el río Singuil (177\% de incremento) comparados con la zona limnética. En cuanto a las variables fisicoquímicas, resultó una asociación positiva con $\mathrm{DBO}_{5}$ y $\mathrm{OD}$, destacándose un aumento significativo del $11 \%$ en las abundancias por incremento en una unidad medida del OD. Por otra parte, $\mathrm{CE} \mathrm{y} \mathrm{NH}_{4}^{+}$dependieron de la temporada y del mes, de tal manera que no pueden interpretarse independientemente. En la segunda temporada la CE se asoció positivamente con las abundancias del dinoflagelado (incremento de densidades de $2 \%$ ) pero negativamente con agosto (decrecimiento del $4 \%$ comparado a marzo). Con respecto al amonio, se destacó una disminución en las abundancias de más del $1000 \%$ en noviembre a incrementos de una unidad de $\mathrm{NH}_{4}^{+}$(Tabla 6). 


\section{S. N. Martínez de Marco et al. - Fitoplancton del embalse Escaba}

Tabla 5. Diversidad especifica (Índice de Shannon-Weaver).

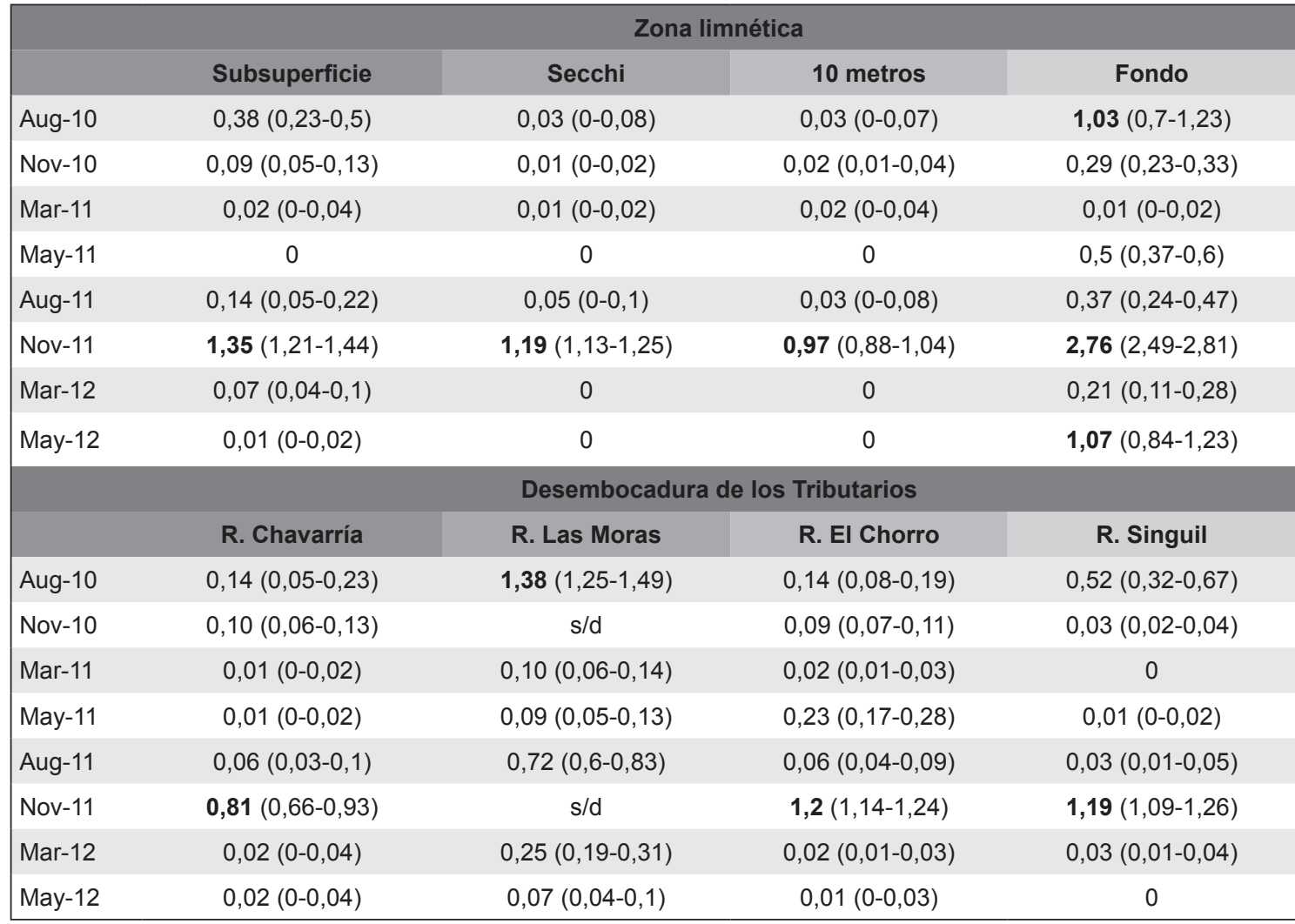

Referencias: Entre paréntesis se señala el intervalo de confianza calculado por técnica Bootstrap. Se resaltan los valores significativos $(p<0,05)$.

\section{Discusión}

La composición taxonómica del embalse Escaba tuvo un considerable número de especies destacándose las diatomeas sobre otros grupos algales. Al comparar nuestros resultados, en general, no fueron coincidentes con otros embalses de Tucumán en los que estuvieron en primer término las algas verdes (Seeligmann \& Tracanna, 1994, 2009; Locascio de Mitrovich et al., 1997; Tracanna et al., 1999, 2000, 2006, 2014a; Martínez De Marco \& Tracanna, 2001; Seeligmann et al., 2001). Salusso \& Moraña (2014) en su inventario de especies del fitoplancton de los embalses Cabra Corral y El Tunal (Salta) también hicieron referencia a las clorofitas como grupo sobresaliente de un total de 334 especies. En el embalse Yacyretá (ArgentinaParaguay), Meichtry de Zaburlín et al. (2013) observaron una situación semejante al reservorio estudiado en este trabajo.
En Escaba sobresalió la presencia prácticamente constante de $C$. hirundinella. Esta especie planctónica, dulceacuícolay alóctona es característica de ambientes meso-eutróficos y suele originar floraciones, colorear el agua y transmitirle olor desagradable e incluso puede provocar mortandad de peces (Boltovskoy, 2005). Su dominancia se asoció a condiciones indeseables en la calidad del agua, ya que el exceso de este dinoflagelado produce alteraciones ecosistémicas que afectan la integridad biológica del ambiente y reducen el valor del reservorio como fuente de recreación. Las mayores densidades de $C$. hirundinella, en general, se dieron a temperaturas altas (superiores a $20^{\circ} \mathrm{C}$ ) similares a las mencionadas como óptimas para su crecimiento en los lagos de la zona templada $\left(12-23^{\circ} \mathrm{C}\right)$ y se asemejaron a lo encontrado para los embalses Cúber y Gorg Blau de España (Moyá \& Ramón, 1984; Ramón \& Moyá, 1984). 
Tabla 6. Modelo lineal generalizado con distribución binomial negativa para evaluar los cambios en la abundancia de Dinophyta (20102012). * Significancia $p<0,05$.

\begin{tabular}{|c|c|c|}
\hline Variable & $\begin{array}{l}\text { Coeficiente } \\
\text { estimado }\end{array}$ & $\begin{array}{l}\text { Error } \\
\text { estándar }\end{array}$ \\
\hline Constante & $4,5^{*}$ & 1,62 \\
\hline $\begin{array}{l}\text { agosto } 2011 \text { - mayo } \\
2012 \text { (temporada 2) }\end{array}$ & $-1,66^{*}$ & 0,76 \\
\hline Río Las Moras & $-0,42$ & 0,23 \\
\hline Río El Chorro & 0,32 & 0,23 \\
\hline Río Singuil & $1,02^{*}$ & 0,28 \\
\hline Río Chavarría & $-0,66^{*}$ & 0,24 \\
\hline mayo & 2,89 & 2,02 \\
\hline agosto & $4,56^{*}$ & 1,69 \\
\hline noviembre & 1,36 & 3,89 \\
\hline $\mathrm{DBO}_{5}$ & $0,009^{*}$ & 0,004 \\
\hline CE & 0,01 & 0,01 \\
\hline $\mathrm{NH}_{4}^{+}$ & $1,07^{*}$ & 0,26 \\
\hline OD & $0,10^{*}$ & 0,05 \\
\hline mayo: CE & $-0,03$ & 0,02 \\
\hline agosto: CE & $-0,04^{*}$ & 0,01 \\
\hline noviembre: CE & $-0,01$ & 0,02 \\
\hline mayo: $\mathrm{NH}_{4}^{+}$ & 31,67 & 19,62 \\
\hline agosto: $\mathrm{NH}_{4}^{+}$ & $-11,12$ & 10,26 \\
\hline noviembre: $\mathrm{NH}_{4}^{+}$ & $-28,27^{*}$ & 2,68 \\
\hline Temporada2: CE & $0,02^{*}$ & 0,006 \\
\hline Theta & 13,04 & 3,24 \\
\hline $\mathrm{AIC}$ & 563,76 & \\
\hline 2.log-verosimilitud & $-521,76$ & \\
\hline
\end{tabular}

Esta Dinophyta puede crecer en aguas con bajas concentraciones de nutrientes ya que logra retenerlos y almacenarlos o migrar en profundidad en su búsqueda (Moyá \& Ramón, 1984). En Escaba durante la primavera de 2010, se registraron elevadas densidades del dinoflagelado a valores bajos de amonio lo que podría deberse a su consumo como nutriente. El MLG corroboró la relación negativa entre el dinoflagelado y el amonio, el cual detectó más del $1000 \%$ en la disminución de las abundancias a incrementos del $\mathrm{NH}_{4}^{+}$particularmente en primavera de 2011.

Además, en noviembre de 2011 la ausencia de C. hirundinella fue acompañada por un aumento considerable de algas verdes en un corto período de tiempo, lo que podría atribuirse a una baja densidad del zooplancton, de manera que disminuye el pastoreo sobre estas microalgas favoreciendo su desarrollo (Bustamante Gil et al., 2012).

Con respecto a los valores de densidad de $C$. hirundinella, Moyá \& Ramón (1984) registraron un máximo de $47 \mathrm{ind} / \mathrm{ml}$ en reservorios de España durante agosto y setiembre de 1978, mientras que el mayor número de individuos obtenido en este trabajo correspondió a $6922 \mathrm{ind} / \mathrm{ml}$ en el río Singuil. En otros ambientes eutróficos las abundancias registradas fueron superiores a las nuestras, por ejemplo en Argentina (embalse La Quebrada, Córdoba) C. hirundinella llegó a 3,25.10 $13 \mathrm{ind} / \mathrm{ml}$. También en Sudáfrica, en un reservorio subtropical, la densidad de las especies de Ceratium varió de $1,5.10^{11}$ a $1,32.10^{12} \mathrm{ind} / \mathrm{ml}$ (Silva et al., 2012).

En el diagrama de Olmstead-Tukey se evidenció además de la dominancia del dinoflagelado que un $14 \%$ de las especies registradas en el estudio cuantitativo fueron dominantes, el $8 \%$ constantes, en general comunes a todas las muestras, el $19 \%$ correspondió a las ocasionales y 59\% a las raras. Esto permitió corroborar una situación especial en la segunda temporada, donde se registró una diferenciación estacional ocasionada por estos dos últimos grupos que sobre todo aparecieron en la primavera de 2011 vinculado a la ausencia de $C$. hirundinella. Estos organismos oportunistas que tienen un rápido crecimiento y reproducción, como las algas verdes de tamaño pequeño, se vieron favorecidos por un incremento de la temperatura y la radiación solar (De León \& Chalar, 2003).

Por otro lado, el MLG ajustado detectó diferencias en los cambios de abundancias de $C$. hirundinella a nivel espacial y temporal, particularmente entre tributarios y la zona limnética (negativamente con el río Chavarría y positivamente con el río Singuil). Probablemente esto se deba a la posición de los tributarios y a la influencia de los vientos S-SE por lo que se destaca un $177 \%$ de incremento en las abundancias en Singuil (al Sur) y un decrecimiento del $48 \%$ en Chavarría (al Norte). En otros embalses también se notó una diferenciación espacial entre las densidades de la zona limnética y las "colas" del reservorio (Kawabata \& Kagawa, 1988; Bustamante Gil et al., 2012). Esta situación podría atribuirse a que los embalses y sus tributarios están sometidos a una dinámica de crecientes y retracciones estacionarias e interanuales que 


\section{S. N. Martínez de Marco et al. - Fitoplancton del embalse Escaba}

provocarían cambios en la disponibilidad de nutrientes y luz entre los lugares de muestreo. Con respecto a los cambios temporales, en los muestreos realizados se observaron mayores abundancias significativas de $C$. hirundinella a incrementos de CE durante la segunda temporada y esta variable mostró una diferencia de un $4 \%$ de decrecimiento en las densidades en agosto (aguas bajas) comparadas con marzo (aguas altas).

Durante mucho tiempo, la evaluación de la calidad en lagos y embalses se centró en parámetros fisicoquímicos como los nutrientes, perfiles de oxígeno, entre otros y actualmente se incorporó la biomasa del fitoplancton expresada en términos de clorofila $a$. Esta última serviría como un estimador de eutrofia de uso muy extendido en varios esquemas de clasificación trófica donde si el máximo de concentración en aguas superficiales es superior a $75 \mu \mathrm{g} / 1$ indicaría una categoría hipereutrófica (OCDE, 1982). Esta condición se observó en Escaba, aunque los valores de clorofila $a$ fueron muy variables a nivel espacial y temporal, destacándose un valor promedio total de biomasa de $419 \mu \mathrm{g} / \mathrm{l}$. Tanto en la zona limnética como en el río Chavarría el registro medio fue inferior (110-291 $\mu \mathrm{g} / \mathrm{l})$ mientras que en los ríos Las Moras, El Chorro y Singuil lo superaron ampliamente (555-719 $\mu \mathrm{g} / \mathrm{l})$. Basándonos en la elevada biomasa registrada en este embalse se podría establecer que su potencial ecológico es deficiente o malo. Asimismo, estos datos difieren notoriamente de los mencionados para otros cuerpos de agua artificiales, incluso si se comparan con los de Tucumán. En estos ambientes lénticos, los registros máximos llegaron a $31 \mu \mathrm{g} / 1$ en La Angostura (Tafí del Valle), $34 \mu \mathrm{g} / 1$ en Río Hondo y $63 \mu \mathrm{g} / \mathrm{l}$ en Dr. C. Gelsi (Tracanna et al., 2014a). Una situación semejante a la de Escaba, donde el principal responsable de los altos valores de clorofila $a$ fue $C$. hirundinella con registros de $250 \mu \mathrm{g} / \mathrm{l}$, fue observada por Mariñelarena et al. (2016) en el embalse Río Tercero (Córdoba). La biomasa se correlacionó significativamente con un gran número de variables bióticas y abióticas, tuvo una relación directa con la temperatura la que es relevante cuando se evalúa la calidad de agua de un reservorio. Este parámetro físico favorece el proceso de eutrofización al producir un incremento en la tasa de reconversión de nutrientes y por lo tanto un aumento de la productividad del lago (Ledesma et al., 2013). La clorofila $a$ además se correlacionó con fósforo y nitrógeno totales, similar a lo citado para algunas represas de Córdoba por Amé et al. (2003), Bazán et al. (2005) y Ledesma et al. (2013). En el caso del nitrato se observó una relación significativa negativa con la concentración de clorofila $a$, lo que podría estar asociado a su consumo cuando existe una proliferación de Ceratium de acuerdo a lo citado por Ferral (2013) para el embalse San Roque, Córdoba.

Coincidente con el predominio de C. hirundinella en la mayoría de los sitios y meses muestreados, la diversidad baja detectada en el embalse Escaba (salvo zona limnética-fondo, primavera de 2011) indicaría una alteración de la calidad del ambiente acuático que condujo a que este dinoflagelado fuese el principal componente del fitoplancton. El fósforo como limitante con una relación N/P en general mayor a 17 (Tracanna et al., 2014b) sería uno de los causantes del crecimiento del dinoflagelado que puede perdurar en condiciones desfavorables por la producción de quistes. Otros factores que favorecerían su permanencia en el embalse estarían vinculados a condiciones de aguas relativamente calmas, a su capacidad de moverse y mantenerse en la columna de agua y de resistir la holomixis. Esto último fue observado también por varios investigadores en otros reservorios de zonas tropicales (Townsend \& Luong-Van, 1998; Townsend, 2001; López et al., 2012). Además, como las células de Ceratium son de mayor tamaño que las de otros fitopláncteres, esto contribuiría a que su herbivoría sea menor. Morales (2016) en un estudio realizado en la represa de La Angostura (Cochabamba, Bolivia) comprobó lo expuesto anteriormente donde algas de dimensiones menores eran presas del zooplancton en lugar de Ceratium furcoides. El gran aporte cuantitativo de $C$. hirundinella en Escaba influyó en la diversidad de forma negativa y altamente significativa.

Con respecto al índice sapróbico, el embalse Escaba quedó definido como polisapróbico dominado por $C$. hirundinella, mientras que en la primavera de 2011, donde se destacaron las algas verdes las aguas fueron de tipo $\alpha$-mesosapróbica. En general, los otros reservorios de Tucumán se clasificaron como $\beta$ a $\beta / \alpha$-mesosapróbicos predominando $A$. granulata en el caso del embalse C. Gelsi y las Chlorophyta y Cyanobacteria en el de Río Hondo (Tracanna et al., 2014a). En el embalse La Angostura si bien no se empleó la valoración de un índice de saprobicidad, se definió la calidad del agua como oligo-mesotrófica en términos de OD, 
$\mathrm{DBO}_{5} \mathrm{y} \mathrm{NH}_{4}^{+}$(Seeligmann \& Tracanna, 2009).

Los resultados de este estudio demostraron la capacidad del fitoplancton para determinar el estado trófico acuático y la caracterización limnológica del embalse. Como consecuencia de la proliferación de C. hirundinella a lo largo del período estudiado, se pudo constatar un significativo deterioro de la calidad de las aguas en base a algunas características organolépticas observadas in situ, elevada biomasa, baja diversidad específica y alta saprobicidad. No obstante durante la primavera del 2011, se observó una leve mejoría del estado trófico del embalse debido a la dominancia de las algas verdes. Por último, este trabajo contribuyó a ampliar el área de distribución del dinoflagelado en la provincia de Tucumán.

Una gestión efectiva es necesaria para mejorar la calidad de este recurso acuático, que tenga en cuenta monitoreos multidisplinarios periódicos a fin de disminuir la eutrofización de este sistema lenítico.

\section{Agradecimientos}

A la Facultad de Ciencias Naturales e IML y a la Fundación Miguel Lillo por sus colaboraciones en los trabajos de campo. Asimismo, este trabajo se realizó en el marco del Proyecto PIUNT 26G/529 subsidiado por la Secretaría de Ciencia, Arte e Innovación Tecnológica (SCAIT) de la UNT.

\section{Bibliografía}

AKAIKE, H. 1973. Information theory and an extension of the maximum likelihood principle. In: PETROV, B. N. \& F. CSAKI (eds.), Proceedings of the Second International Symposium on Information Theory, pp. 267-281. Akademiai Kiado, Budapest.

AMÉ, M. V., M. DEL PILAR DÍAZ \& D. A. WUNDERLIN. 2003. Occurrence of toxic cyanobacterial blooms in San Roque Reservoir (Córdoba, Argentina): a field and chemometric study. Environ. Toxicol. 18: 192-201.

BAZÁN, R., M. CORRAL, M. PAGOT, A. RODRÍGUEZ, N. RODRÍGUEZ, N. LARROSA, A. COSSAVELLA, S. OLMO, E. BONFANTI \& F. BUSSO. 2005. Teledetección y modelado numérico para el análisis de la calidad de agua del embalse Los Molinos. Ing. Hidráulica en México 20: 121-135.

BEAUJEAN, A. A. \& G. B. MORGAN. 2016. Tutorial on using regression models with count outcomes using R. Prac. Assess. Res. Eval. 21: 1-19.
BOLTOVSKOY, A. 2005. Ceratium hirundinella Schrank: Un dinoflagelado invasor en lagos y embalses. Bol. Soc. Argent. Bot. 40: 9.

BUSTAMANTE GIL, C., J. J. RAMÍREZ RESTREPO, A. BOLTOVSKOY \& A. VALLEJO. 2012. Spatial and temporal change characterization of Ceratium furcoides (Dinophyta) in the equatorial reservoir Riogrande II, Colombia. Acta Limnol. Bras. 24: 207-219.

CONZONNO, V. 2009. Limnología química. $1^{\mathrm{a}} \mathrm{ed}$ Editorial de la Universidad Nacional de La Plata, La Plata.

DE LEÓN, L. \& G. CHALAR. 2003. Abundancia y diversidad del fitoplancton en el Embalse de Salto Grande (Argentina - Uruguay). Ciclo estacional y distribución espacial. Limnetica 22: 103-113.

DEL GIORGIO, P. A., A. L. VINOCUR, R. J. LOMBARDO \& G. TELL. 1991. Progressive changes in the structure and dynamics of the phytoplankton community along a pollution gradient in a lowland river - a multivariate approach. Hydrobiologia 224: 129-154.

DI RIENZO, J. A., F. CASANOVES, M. G. BALZARINI, L. GONZALEZ, M. TABLADA \& C. W. ROBLEDO. 2013. InfoStat versión 2013. Grupo InfoStat, FCA, Universidad Nacional de Córdoba, Argentina. Disponible en: http://www.infostat.com.ar.

FERNÁNDEZ, D. S., M. E. PUCHULU \& M. CZERNICZYNIEC. 2007. Trabajo de campo en el embalse Escaba. En: CICERONE, D. S. \& M. DEL V. HIDALGO (eds.), Los Humedales de la Cuenca del Rio Sali, pp. 227-254. Jorge Baudino Ediciones, Buenos Aires.

FERRAL, A. 2013. Análisis espacio-temporal del efecto del sistema de aireación artificial en el Embalse San Roque. Integración de datos de campo y técnicas geoespaciales. Tesis de Maestría. Facultad de Matemática, Astronomía y Física, UNC.

HAMM,A. 1969. Die Ermittlung der Gewässergüteklassen bei Fließgewässem nach den Gewässergütesystem und Gewässergütenomogramm. Münch. Beitr. Abwass. Fisch. Flussbiol. 15: 46-48.

HUSZAR, V. L. M., L. H. S. SILVA, P. DOMINGOS, M. M. MARINHO \& S. MELO. 1998. Phytoplankton species composition is more sensitive than OECD criteria to the trophic status of three Brazilian lakes. Hydrobiologia 369/370: 59-71.

KAWABATA, Z. \& H. KAGAWA. 1988. Distribution pattern of the dinoflagellate Ceratium hirundinella (O. F. Müller) Bergh in a reservoir. Hydrobiologia 169: 319-325.

LEDESMA, C., M. BONANSEA, C. M. RODRÍGUEZ \& A. R. SÁNCHEZ DELGADO. 2013. Determinación de indicadores de eutrofización en el embalse Río Tercero, Córdoba (Argentina). Rev. Ciên. Agron. 44: 419-425. 


\section{S. N. Martínez de Marco et al. - Fitoplancton del embalse Escaba}

LOCASCIO DE MITROVICH, C., A. VILLAGRA DE GAMUNDI, B. TRACANNA, C. SEELIGMANN \& C. BUTI. 1997. Situación actual de la problemática limnológica de los embalses de la provincia de Tucumán (Argentina). Lilloa 39: 81-93.

LOEZ, C. R. 1995. Determinación de clorofila $a$. En: LOPRETTO, E. C. \& G. TELL (eds.), Ecosistemas de aguas continentales. Metodología para su estudio I, pp. 263-269. Ediciones Sur, La Plata.

LÓPEZ, N. L., C. A. RIVERA RONDÓN, Á. ZAPATA, J. JIMÉNEZ, W. VILLAMIL, G. ARENAS, C. RINCÓN \& T. SÁNCHEZ. 2012. Factors controlling phytoplankton in tropical high-mountain drinkingwater reservoirs. Limnetica 31: 305-322.

MAGURRAN, A. E. 2004. Measuring biological diversity. Blackwell Publishing, Oxford.

MARGALEF, R. 1983. Limnología. $1^{\circ}$ ed. Omega, Barcelona.

MARIÑELARENA, A., M. E. MAC DONAGH, J. DONADELLI \& M. A. CASCO. 2016. Un caso inusual de eutrofización en el Embalse Río Tercero: el posible rol de dos bioinvasores. Biol. Acuát. 31: 10-18.

MARTÍNEZ DE MARCO, S. N. \& B. C. TRACANNA. 2001. Limnología del embalse Dr. C. Gelsi (El Cadillal) Tucumán - Argentina. IV: Cambios espaciales y temporales del fitoplancton. Lilloa 40: 223-234.

MCCULLAGH, P. \& J. A. NELDER. 1983. Generalized linear models. Chapman and Hall, London.

MEICHTRY DE ZABURLÍN, N., R. E. VOGLER, V. M. LLANO \& I. S. M. MARTENS. 2013. Fitoplancton del embalse Yacyretá (Argentina-Paraguay) a una década de su llenado. Rev. Mex. Biodiv. 84: 225-239.

MORALES, E. A. 2016. Floración de Ceratium furcoides (Levander) Langhans (Dinoflagellata, Dinophyceae) en la represa de La Angostura, Cochabamba, Bolivia. Acta Nova 7: 389-398.

MOYÁ, G. \& G. RAMÓN. 1984. Variación espaciotemporal de Ceratium hirundinella, en los embalses de Cúber y Gorg Blau (Mallorca). Limnetica 1: 285-290.

NAVARRO, E., E. GARCÍA-BERTHOU \& J. ARMENGOL. 2010. La calidad ecológica de los embalses. I y $C$ 401: 80-87.

OCDE (Organización para la Cooperación y el Desarrollo Económico). 1982. Eutrophication of Waters. Monitoring, Assessment and Control. Cooperative Programmers on Monitoring of Inland Waters (Eutrophication Control), Environment Directorate, OCDE Paris, Final Report. France.

PANTLE, R. \& H. BUCK. 1955. Die biologisch Überwachung der Gewässer und die Darstellung der Egerbnisse. Gas. Wasserfach. 96: 604-607.

PANTORRILLA, M. M. \& V. A. NÚÑEZ REGUEIRO. 2006. Investigaciones arqueológicas en la zona de Escaba, provincia de Tucumán: asentamientos Condorhuasi y Aguada en las Yungas. Intersecciones Antro. 7: 235-245.
RAMÓN, G. \& G. MOYÁ. 1984. Distribución estacional de Planctonema lauterbornii (Ulotrichaceae) en dos embalses de aguas mineralizadas (Cúber y Gorg Blau, Mallorca). Limnetica 1: 291-296.

R DEVELOPMENT CORE TEAM. 2005. $R$ : $A$ language and environment for statistical computing. R Foundation for Statistical Computing, Vienna, Austria. ISBN 3-900051-07-0, Disponible en: http:// www.R-project.org.

REARTE, J. 1981. Provincia de Tucumán. En: Centro Editor de América, org., Atlas Físico de la República Argentina I, pp. 99-105. Buenos Aires.

REYNOLDS, C. S. 1998. What factors influence the species composition of phytoplankton in lakes of different trophic status? Hydrobiologia 369/370: 1126.

RODRÍGUEZ, M. I., M. A. BUSTAMANTE, A. L. RUIBAL, M. A. RUÍZ \& F. BUSSO. 2000. Estudio del fitoplancton en el marco de monitoreo del Embalse San Roque (Cba.). IV Congreso Argentino de Limnología. Instituto Nacional del Agua. S. C. de Bariloche. 14 pp.

ROJO, C. 1998. Differential attributes of phytoplankton across the trophic gradient: a conceptual landscape with gaps. Hydrobiologia 369/370: 1-9.

ROTT, E. 1988. Some aspects of the seasonal distribution of flagellates in mountain lakes. Hydrobiologia 161: 159-170.

SALUSSO, M. M. \& L. B. MORAÑA. 2014. Fitoplancton de embalses subtropicales del noroeste argentino. Gest. Ambient. 17: 209-222.

SANTILLÁN DE ANDRÉS, S. E. \& T. R. RICCI. 1980. Geografia de Tucumán (Consideraciones generales). Editorial de la Universidad Nacional de Tucumán. Publicación 1299, Tucumán.

SEELIGMANN, C. T. \& B. C. TRACANNA. 1994. Limnología del embalse El Cadillal (Tucumán, Argentina) II. Estudio cualitativo del fitoplancton. Cryptogamie Algol. 15: 19-35.

SEELIGMANN, C. \& B. C. TRACANNA. 2009. Dinámica del fitoplancton en un embalse de alta cota del Noroeste Argentino (Tucumán). Limnetica 28: 105-124.

SEELIGMANN, C., B. C. TRACANNA, S. MARTÍNEZ DE MARCO \& S. ISASMENDI. 2001. Algas fitoplanctónicas en la evaluación de la calidad de agua de sistemas lóticos en el Noroeste Argentino. Limnetica 20: 123-133.

SEIP, K. L. \& C. S. REYNOLDS. 1995. Phytoplankton functional attributes along trophic gradient and season. Limnol. Oceonogr. 40: 589-597.

SILVA, L. C., I. CARNIELLI LEONE, M. J. SANTOSWISNIEWSKI, A. CARVALHO PERET \& O. ROCHA. 2012. Invasion of the dinoflagellate Ceratium furcoides (Levander) Langhans 1925 at tropical reservoir and its relation to environmental variables. Biota Neotrop. 12: 93-100. 
SIROMBRA, M. 2003. Viaje de campo dique Escaba. Guía de apoyo de la Cátedra de Ecología General. FCN e IML, UNT. 12 pp.

SOKAL, R. R. \& F. J. ROHLF. 1981. Biometry the principles and practice of statistics in biological research. $2^{\text {nd }}$ ed. WH Freeman and Company, San Francisco.

TOWNSEND, S. A. 2001. Perennial domination of phytoplankton by Botryococcus and Peridinium in a discontinuously polymictic reservoir (tropical Australia). Arch. Hydrobiol. 151: 529-548.

TOWNSEND, S. A. \& J. T. LUONG-VAN. 1998. Phytoplankton biomass and composition in Manton River Reservoir, a mesotrophic impoundment in the Australian wet/dry tropics. Int. Rev. Hydrobiol. 83: $113-120$

TRACANNA, B. C., C. T. SEELIGMANN, V. MIRANDE, L. B. DE PARRA, M. T. DE PLAZA \& F. M. MOLINARI. 1999. Cambios espaciales y temporales del fitoplancton en el embalse Río Hondo (Argentina). Bol. Soc. Argent. Bot. 34: 101105.

TRACANNA, B. C., S. MARTÍNEZ DE MARCO, C. SEELIGMANN, S. ISASMENDI \& A. ELÍAS. 2000. Distribución vertical del fitoplancton del embalse Río Hondo, Argentina. En: FARÍAS, H. D., M. T. PILÁN, M. J. BORSELLINO, F. J. PECE, A. DEL R. STORNIOLO \& M. T. MATTAR (eds.), Uso y preservación de los recursos hídricos en los umbrales del siglo XXI. Memorias del XVIII Congreso Nacional del Agua, Santiago del Estero.

TRACANNA, B. C., S. N. MARTÍNEZ DE MARCO, M. J. AMOROSO, N. ROMERO, P. CHAILE \& A. MANGEAUD. 2006. Physical, chemical and biological variability in the Dr. C. Gelsi reservoir (NW Argentine): A temporal and spatial approach. Limnetica 25: 787-808.
TRACANNA, B. C., C. T. SEELIGMANN, V. MIRANDE, S. N. MARTÍNEZ DE MARCO \& S. ISASMENDI. 2014a. Peri-Pampean Sierras aquatic systems in Tucumán Province. In: TELL, G., I. IZAGUIRRE \& I. O'FARRELL (eds.), Freshwater phytoplankton of Argentina. Phytoplankton diversity and ecology in different aquatic systems. Advanc. Limnol. 65, pp. 199-213. Editorial E. Schweizerbart Science Publishers, Stuttgart.

TRACANNA, B. C., S. N. MARTÍNEZ DE MARCO, M. DE LOS Á. TABOADA, V. MIRANDE, M. DE L. GULTEMIRIAN \& S. C. ISASMENDI. 2014b. Assessment of the physicochemical variables of a subtropical reservoir in the northwest of Argentina. Acta Limnol. Bras. 26: 367-380.

TUNDISI, J. G. 2008. Recursos hídricos no futuro: problemas e soluções. Est. Av. 22: 7-16.

UTERMÖHL, H. 1958. Zur Vervollkommung der Quantitativen Phytoplankton Methodik. Mitt. Int. Verein. Limnol. 9: 1-38.

WESTERN, D. 2001. Human-modified ecosystems and future evolution. Proc. Natl. Acad. Sci. U.S.A. 98: 5458-5465.

Recibido el 8 de mayo de 2018, aceptado el 4 de octubre de 2018. Editora: Luz Allende. 